OPEN ACCESS

Edited by:

Pantelis Theodoros Nikolaidis, University of West Attica, Greece

Reviewed by:

Nuno Domingos Garrido,

University of Trás-os-Montes and Alto Douro, Portugal Milivoj Dopsaj,

University of Belgrade, Serbia Mustafa Sögüt,

Middle East Technical

University, Turkey

*Correspondence: Jorge E. Morais morais.jorgestrela@gmail.com

Specialty section:

This article was submitted to

Exercise Physiology,

a section of the journal

Frontiers in Physiology

Received: 07 April 2021

Accepted: 17 August 2021

Published: 16 September 2021

Citation:

Morais JE, Barbosa TM, Forte $P$, Silva AJ and Marinho DA (2021) Young Swimmers' Anthropometrics,

Biomechanics, Energetics, and Efficiency as Underlying Performance

Factors: A Systematic Narrative Review. Front. Physiol. 12:691919. doi: 10.3389/fphys.2021.691919

\section{Young Swimmers' Anthropometrics, Biomechanics, Energetics, and Efficiency as Underlying Performance Factors: A Systematic Narrative Review}

\author{
Jorge E. Morais ${ }^{1,2 *}$, Tiago M. Barbosa ${ }^{1,2}$, Pedro Forte ${ }^{1,2,3}$, António J. Silva ${ }^{2,4}$ and \\ Daniel A. Marinho ${ }^{2,5}$
}

\begin{abstract}
'Department of Sport Sciences, Instituto Politécnico de Bragança, Bragança, Portugal, ${ }^{2}$ Research Center in Sports Health and Human Development (CIDESD), University of Beira Interior, Covilhã, Portugal, ${ }^{3}$ Department of Sports, Higher Institute of Educational Sciences of the Douro, Penafiel, Portugal, ${ }^{4}$ Department of Sport Sciences, University of Trás-os-Montes and Alto Douro, Vila Real, Portugal, ${ }^{5}$ Department of Sport Sciences, University of Beira Interior, Covilhã, Portugal
\end{abstract}

Introduction: In youth swimming, researchers are interested in understanding how anthropometry and parameters related to swimming technique (biomechanics, energetics, and efficiency) influence the performance. However, there is not any review in the literature that consolidates the body of knowledge of this topic. The objective of this study was to review systematically the current body of work on the influence of determinant factors related to swimming technique (biomechanics, energetics, and efficiency) and anthropometry in the young performance of swimmers.

Methods: The Preferred Reporting Items for Systematic Reviews and Meta-Analyses (PRISMA) guidelines were used to identify relevant studies.

Results: After screening, 240 studies were analyzed and 59 related to swimming performance, and its determinant factors were retained for synthesis. Studies revealed a high-quality index by PEDro scale (mean score was $7.17 \pm 1.40$ ). Twenty-five studies were longitudinal designs and the remaining 34 cross-sectional designs. Most of the studies ( $N=39,66.1 \%$ ) reported concurrently two or more determinant factors (anthropometrics, biomechanics, energetics, and efficiency).

Conclusion: Youth swimming research relies on a multifactorial assessment. From the synthesis, it is possible to conclude that the performance of young swimmers is characterized by a multifactorial, holistic, and dynamic phenomenon. Better performance has always been related to better swimming technique and higher anthropometrics. This suggests that both anthropometrics (i.e., nature) and training (i.e., nurture) play key roles in the swimming performance of young swimmers.

Keywords: talent, identification, development, swimming, determinants, sports career 


\section{INTRODUCTION}

One of the major topics of interest in sports science is the identification of talented young athletes. This process is based on talent identification and development (TID) programs that aim to identify young athletes with potential for success in adult/elite sport (Blume and Wolfarth, 2019). Detecting talent at an early stage is considered a key factor in increasing a chance of a country of achieving success in sports (Vaeyens et al., 2009). Competitive swimming is one of the three main modern Olympic sports. In competitive swimming, Olympic, and World records are broken on a regular basis, challenging the limits of athletes. Practitioners and researchers are eager to predict the next top-ranked swimmer who will contribute to the superiority of their country at major international competitions.

Talent identification and development programs follow standard steps: (1) identifying the athletes with the potential to deliver the best performances in adulthood and determining the variables responsible for such performances; (2) understanding the development and changes in performance and its determinant factors, according to a training program, and; (3) following up in order to allow to understand the variation of such variables and its relationship with performance over a given time (Morais et al., 2017). To get deeper insights into how determinant factors of swimmers change over time, their interaction and their effect on performance, researchers, and coaches should focus on a long-term approach (Staub et al., 2020a; Zacca et al., 2020). Long-term athlete development (LTAD) programs focus on providing young athletes with fundamental motor skills in tandem to their maturation stage (Martindale et al., 2005; Lang and Light, 2010).

Literature reports that performance in youth swimming is highly dependent on variables related to technique (i.e., nurture) and body dimensions (i.e., nature) (Abbott et al., 2021). Thus, research on young swimmers has been largely focused on the assessment of anthropometrics (Geladas et al., 2005; Nevill et al., 2020), strength and conditioning (Garrido et al., 2010b; Amaro et al., 2017), biomechanics (Morais et al., 2012; Silva et al., 2012), energetics, and efficiency (Denadai et al., 2000; Toubekis et al., 2006), as well as interactions among some or all of them (Morais et al., 2017; Barbosa et al., 2019). Nonetheless, most of these are cross-sectional designs. Such research design does not provide substantial information on the dynamic and complex interactions among the performance determinants over time (Morais et al., 2017). Conversely, longitudinal designs can help gather information on: (1) how determinant factors interplay and affect performance; (2) the dynamic changes that take place at these early ages, and; (3) the change of the partial contribution of each determinant factor in the performance over time (Lätt et al., 2009a,b; Morais et al., 2014a). Notwithstanding, in the last decade, it has been suggested that research on sports performance should adopt a multidisciplinary approach to better understand the athlete (Phillips et al., 2010; Seifert et al., 2013). Moreover, the relationship with the environment must be taken into account, as this relationship is considered under a complex and dynamic system framework (Phillips et al., 2010; Seifert et al., 2013). If so, it will be possible to understand the partial contribution of each determinant factor or set of factors in the performance, which will most likely change over time, as aforementioned (Barbosa et al., 2014; Morais et al., 2015).

Literature reports a review study about the relationship between performance and determinant factors in master swimmers (Ferreira et al., 2016). More recently, Koopmann et al. (2020) have systematically reviewed technical skills in talented youth athletes (which included three articles about swimmers). That said, there is no review that consolidates the available evidence of how different determinant factors can affect youth swimming performance. Therefore, the aim of this study was to review the current body of work on the influence of determinant factors related to swimming technique (biomechanics, energetics, and efficiency) and anthropometrics in the performance of young swimmers.

\section{METHODS}

\section{Literature Search and Article Selection}

The Web of Science, PubMed, and Scopus databases were searched to identify studies that aimed to identify, analyze, or predict the performance of young swimmers and its determinant factors (anthropometrics, biomechanics, energetics, and efficiency). These electronic search databases were chosen because they are the most used in sports science. As an initial search strategy, the title, abstract, and the studies keywords were identified and read carefully for a first scan and selection of the journal articles. To search the articles, the following fields were used: (1) Web of Science- "Topic"; (2) PubMed-“All fields"; and (3) Scopus- "Article title, abstract, keywords." A Boolean search strategy was used with the operators AND, OR, and a combination of the keywords presented in Table 1 (whenever suitable). If one of these fields (title, abstract, and keywords) was not clear about the topic under analysis, the complete article was read and fully reviewed to ensure its inclusion or exclusion. After deleting all duplicated and unrelated articles, 59 articles were included. The final search was carried out on March 21, 2021. Table 1 presents the used PI(E)CO search strategy (Ppatient, problem or population; I-intervention; $\mathrm{E}-$ exposure; $\mathrm{C}$-comparison, control, or comparator; $\mathrm{O}$-outcomes).

The inclusion criteria were the following: (1) written in English; (2) published in a peer-reviewed journal; (3) related to assessment of the performance of young swimmers (i.e., race events or swim trials/bouts) and its determinant factors (anthropometrics, biomechanics, energetics, and efficiency); (4) included healthy and able-bodied swimmers, and; (5) reported an average sample age limited to the age of 13 (it is considered that children tend to enter the puberty stage from this age onwards-Mirwald et al., 2002). The exclusion criteria were: (1) studies that included disabled swimmers or with any pathology; (2) review papers, conference papers, and books; (3) studies including animal models; (4) publications not related to the topic in question (e.g., in other scientific fields, such as nutrition, psychology, or any other topic not related to performance); (5) studies that recruited several age groups, but did not clearly report the average of at least an age group of 13 years or under. 
TABLE 1 | PI(E) CO (P-patient, problem or population; I-intervention; E-exposure; C-comparison, control, or comparator; O-outcomes) search strategy.

\begin{tabular}{|c|c|c|c|}
\hline Population & $\begin{array}{l}\text { Intervention or } \\
\text { Exposure }\end{array}$ & $\begin{array}{l}\text { Comparison } \\
\text { (design) }\end{array}$ & Outcome \\
\hline Swimmer ${ }^{*}$ & Talent & Cross-sectional & Performance \\
\hline Athlete $^{*}$ & Identification & Longitudinal & Velocity/speed \\
\hline Youth & Development & Experimental & Length \\
\hline Child $^{*}$ & $\begin{array}{l}\text { Long-term } \\
\text { development }\end{array}$ & Exploratory & Area \\
\hline Boy ${ }^{\star}$ & Anthropometrics & Descriptive & Volume \\
\hline Girl $^{*}$ & Biomechanics & $\begin{array}{l}\text { Randomized } \\
\text { control trial }\end{array}$ & Mass \\
\hline Young & Energetics & & Girth \\
\hline \multirow[t]{26}{*}{ Age-group* } & Efficiency & & Skinfold \\
\hline & Motor control & & Stroke length \\
\hline & $\begin{array}{l}\text { Strength and } \\
\text { conditioning }\end{array}$ & & Stroke frequency \\
\hline & & & Stroke rate \\
\hline & & & $\begin{array}{l}\text { Intra-cyclic variation of } \\
\text { velocity/speed }\end{array}$ \\
\hline & & & Passive drag \\
\hline & & & Active drag \\
\hline & & & Coefficient of drag \\
\hline & & & Oxygen uptake \\
\hline & & & Oxygen consumption \\
\hline & & & Lactate \\
\hline & & & Heart rate \\
\hline & & & Aerobics \\
\hline & & & Anaerobic lactic \\
\hline & & & Anaerobic alactic \\
\hline & & & Energy cost \\
\hline & & & Energy expenditure \\
\hline & & & Propelling efficiency \\
\hline & & & Froude efficiency \\
\hline & & & Stroke Index \\
\hline & & & Critical velocity/speed \\
\hline & & & Index of coordination \\
\hline & & & Strength \\
\hline & & & Maximal strength \\
\hline & & & Power \\
\hline & & & Mechanical power \\
\hline
\end{tabular}

${ }^{\star}$ Truncation to retrieve words with different endings.

Figure 1 depicts the PRISMA flow diagram for identifying, screening, checking eligibility, and inclusion of the articles. There were four articles (Figure 1- "Additional records identified through other sources" that were obtained by submissions reviewed and based on references from the articles retained.

\section{Quality Assessment}

The PEDro scale was used to assess the quality of the selected articles. It was observed that this scale is a suitable and valid tool to assess the methodological quality (de Morton, 2009). Two reviewers read all the included articles and scored them according to the scale items (poor quality if score $\leq 3$; fair quality if the score is between 4 and 5; high quality if the score is between 6 and 10) (de Morton, 2009). Afterwards, the Cohen's Kappa (K) was computed to assess the agreement between reviewers. It was interpreted as: (1) no agreement if $K \leq 0$; (2) none to slight agreement if.01 $<K \leq 0.20$; (3) fair if. $21<K \leq 0.40$; (4) moderate if.41 $<K \leq 0.60$; (5) substantial if. $61<K \leq 0.80$, and; (6) almost perfect if. $81<K \leq 1.00$. Studies were compared based on the: (1) research design (cross-sectional vs. longitudinal designs), and (2) year of publication (published before or in 2010 vs. published after 2010). In both comparisons, distribution was non-normal. Thus, the Mann-Whitney $U$ test $(p \leq 0.05)$ was selected for further inferential analysis.

\section{RESULTS}

PEDro mean score was $7.17 \pm 1.40$ points (i.e., high quality). The Cohen's Kappa yielded an almost perfect agreement between reviewers ( $K=0.937, p<0.001)$. There were non-significant differences in PEDRo scores based on research design $(p=$ $0.651)$, or year of publication ( $p=0.477)$.

Table 2 summarizes the sample demographics, including the sample size, chronological age, maturation stage, years of experience, and competitive level based on FINA points.

Table 3 presents the summary of the studies purpose, research design, type of collected data (anthropometrics, biomechanics, energetics, and efficiency), and performance. Overall, swimming performance (time or speed) was clearly reported (normative data for time or speed at a given distance) in 51 reviewed studies (86.4\%) (Table 3). Out of 59 included studies, 25 (42.4\%) were based on longitudinal designs, and the remaining 34 (57.6\%) were cross-sectional (Table 3). Fifty-four studies (91.5\%) reported anthropometric parameters, including 34 cross-sectional designs and 20 longitudinal designs. Also, 54 studies (91.5\%) analyzed the biomechanics (32 cross-sectional and 22 longitudinal designs), and $42(71.2 \%)$ the energetics and efficiency ( 25 cross-sectional and 17 longitudinal designs) (Table 3). Thirty-nine studies $(66.1 \%)$ reported anthropometrics, biomechanics, energetics and efficiency, and performance concurrently (i.e., interdisciplinary research). Three studies (5.1\%) focused exclusively on tracking the swimming performance from childhood to adulthood.

\section{DISCUSSION}

The aim of this study was to review the current body of work on the influence of determinant factors related to swimming technique and anthropometrics in the performance of young swimmers. It was recognized that the performance of young swimmers is not exclusively dependent on one or a small set of determinant factors related to swimming technique and anthropometrics. It is rather influenced by a multidisciplinary interaction of several determinant factors. Furthermore, these factors and their partial contribution to performance can change over time according to the training plan or designed periodization. 


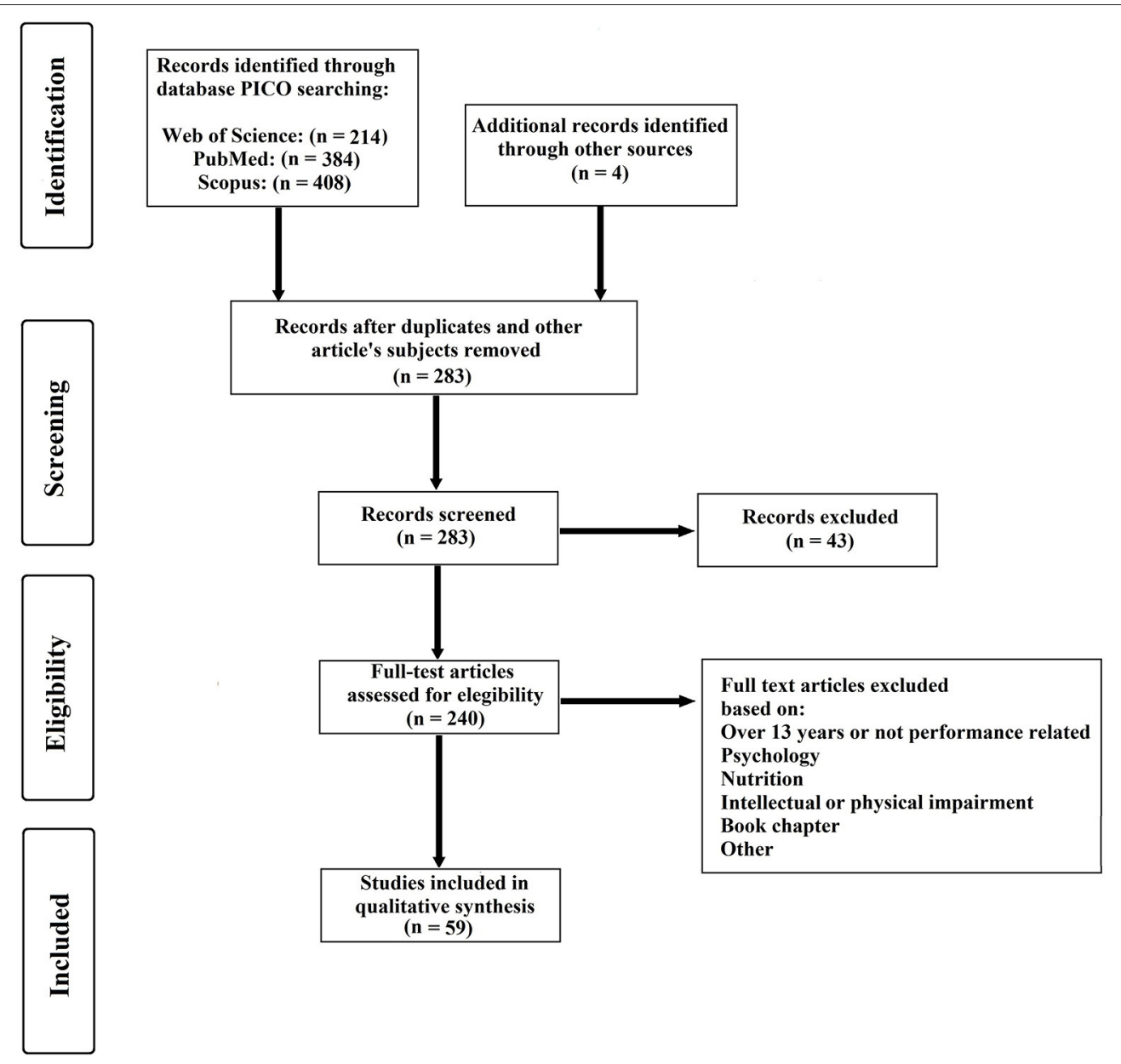

FIGURE 1 | Summary of PRISMA flow for search strategy.

\section{Anthropometrics and Growth}

Most studies ( $\mathrm{N}=55, \sim 93 \%)$ included in this review assessed the anthropometrics. Body dimensions are related to nature, i.e., genetically determined (Saavedra et al., 2010; Majid et al., 2019; Tijani et al., 2019). Researchers are prone to assess the anthropometrics of young swimmers of both sexes, because these features play one of the major roles in the swimming performance, kinematics, energetics, and efficiency (Geladas et al., 2005; Jürimäe et al., 2007; Lätt et al., 2009a), in addition to hydrodynamics (Kjendlie and Stallman, 2008; Barbosa et al., 2014). Cross-sectional studies showed that variables such as height $(\mathrm{H})$, arm span (AS), and hand length (HL) are strongly and positively correlated to Freestyle sprint performance (i.e., 50 or $100 \mathrm{~m}$ ) (Geladas et al., 2005; Morais et al., 2012; Bielec and Jurak, 2019). The same trend was verified in breaststroke, in which swimmers with longer upper-limb lengths and wider girths had a significant advantage (i.e., better performance in the $100 \mathrm{~m}$ ) (Sammoud et al., 2018). In backstroke (25- and 50-m pace), it was observed that postpubertal swimmers were significantly faster than their prepubertal counterparts (Silva et al., 2013). The significant higher body mass (BM), H, and AS shown by the postpubertal swimmers contributed to this (Silva et al., 2013). The same trend was verified in other freestyle distances $(100,200$, and $400 \mathrm{~m}-$ Mezzaroba and Machado, 2014; 50 and $400 \mathrm{~m}-$ Ferraz et al., 2020), in which H, AS/H ratio (Ferraz et al., 2020) and other lengths related to upper- (TUEL) and lower-limbs (TLEL) lengths were significantly longer in mature swimmers (Mezzaroba and Machado, 2014).

Cluster analysis identifies homogeneous subgroups of swimmers within a larger sample (Barbosa et al., 2014; Morais et al., 2015, 2020b). Cluster analysis detects swimmers within a specific cluster that shares similar characteristics but is very different from other swimmers who do not belong to that cluster (Morais et al., 2015). Faster swimmers, competing in the $100-\mathrm{m}$ freestyle, were clustered as a group with larger anthropometric features such as BM, AS, $\mathrm{H}$, chest perimeter $(\mathrm{CP})$, hand surface area (HSA), frontal surface area (FSA), trunk transverse surface area (TTSA), and body surface area (BSA) (Morais et al., 2015, 2020b). A study that aimed to identify key somatic variables in youth swimming recognized that all swimmers benefited from having less body fat (BF), wider shoulders and hips, longer AS, and forearm girth (FG) 
TABLE 2 | The summary of the sample demographics of each study included for analysis.

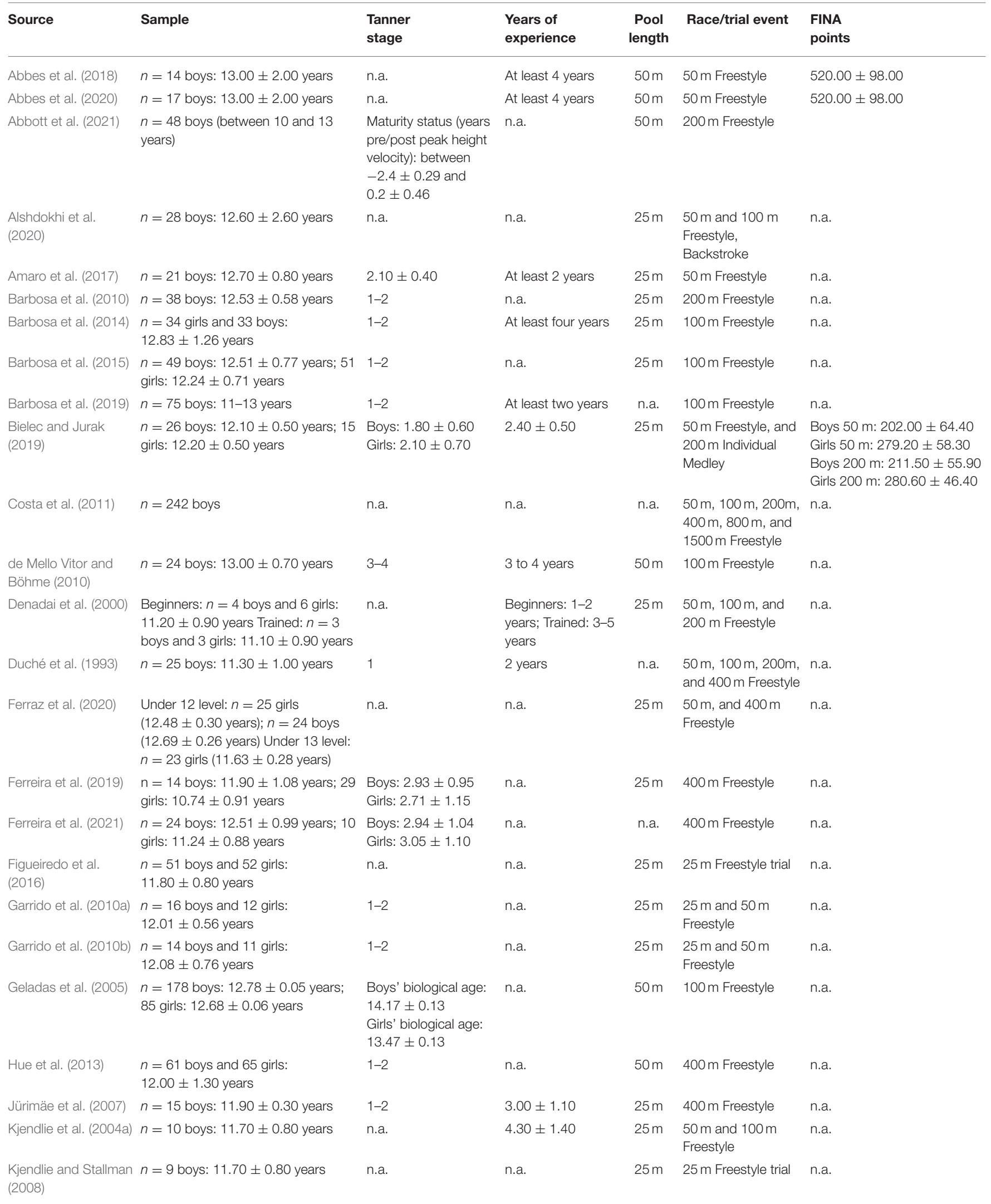


TABLE 2 | Continued

\begin{tabular}{|c|c|c|c|c|c|c|}
\hline Source & Sample & $\begin{array}{l}\text { Tanner } \\
\text { stage }\end{array}$ & $\begin{array}{l}\text { Years of } \\
\text { experience }\end{array}$ & $\begin{array}{l}\text { Pool } \\
\text { length }\end{array}$ & Race/trial event & $\begin{array}{l}\text { FINA } \\
\text { points }\end{array}$ \\
\hline Lätt et al. (2009a) & $n=29$ boys: $13.0 \pm 1.80$ years & $2.30 \pm 1.00$ & $3.00 \pm 1.10$ & $25 \mathrm{~m}$ & 400 m Freestyle & n.a. \\
\hline Lätt et al. (2009b) & $n=26$ girls: $12.70 \pm 2.20$ years & $2.30 \pm 0.80$ & $3.70 \pm 1.00$ & $25 \mathrm{~m}$ & 400 m Freestyle & n.a. \\
\hline Majid et al. (2019) & $n=4$ boys: $11.15 \pm 0.96$ years & n.a. & n.a. & $50 m$ & $50 \mathrm{~m}$ Breaststroke & n.a. \\
\hline Marinho et al. (2011) & $\begin{array}{l}n=12 \text { boys and } 8 \text { girls: } \\
12.10 \pm 0.72 \text { years }\end{array}$ & n.a. & $3.70 \pm 1.26$ & n.a. & $\begin{array}{l}50 \mathrm{~m}, 100 \mathrm{~m} \text {, and } \\
200 \mathrm{~m} \text { Freestyle, } \\
\text { Backstroke, } \\
\text { Breaststroke, } \\
\text { and } \\
\text { Butterfly }\end{array}$ & n.a. \\
\hline Marinho et al. (2020) & $\begin{array}{l}n=75 \text { boys and } 76 \text { girls: } \\
13.02 \pm 1.19 \text { years }\end{array}$ & n.a. & $3.36 \pm 0.77$ years & $25 \mathrm{~m}$ & 100 m Freestyle & n.a. \\
\hline $\begin{array}{l}\text { Mezzaroba and } \\
\text { Machado (2014) }\end{array}$ & $\begin{array}{l}n=13 \text { boys: } 10.70 \pm 0.90 \text { years; } n \\
=11 \text { boys: } 13.00 \pm 0.50 \text { years }\end{array}$ & $\begin{array}{l}2.20 \pm 0.80 \text { and } \\
3.60 \pm 0.80\end{array}$ & $\begin{array}{l}3.50 \pm 1.90 \text { and } \\
5.70 \pm 3.30 \text { years }\end{array}$ & $50 \mathrm{~m}$ & $\begin{array}{l}100 \mathrm{~m}, 200 \mathrm{~m}, \text { and } \\
400 \text { m Freestyle }\end{array}$ & n.a. \\
\hline Morais et al. (2012) & $\begin{array}{l}n=73 \text { boys: } 12.72 \pm 1.03 \text { years; } 64 \\
\text { girls: } 11.47 \pm 0.66 \text { years }\end{array}$ & $1-2$ & n.a. & $25 \mathrm{~m}$ & 100 m Freestyle & n.a. \\
\hline Morais et al. (2013a) & $\begin{array}{l}n=62 \text { boys: } 12.76 \pm 0.72 \text { years; } 64 \\
\text { girls: } 11.89 \pm 0.93 \text { years }\end{array}$ & $1-2$ & n.a. & $25 \mathrm{~m}$ & 100 m Freestyle & n.a. \\
\hline Morais et al. (2013b) & $\begin{array}{l}n=15 \text { boys: } 12.30 \pm 0.63 \text { years; } 18 \\
\text { girls: } 11.77 \pm 0.92 \text { years }\end{array}$ & $1-2$ & $3.18 \pm 0.52$ years & $25 \mathrm{~m}$ & 100 m Freestyle & n.a. \\
\hline Morais et al. (2014a) & $\begin{array}{l}n=14 \text { boys: } 12.33 \pm 0.65 \text { years; } 16 \\
\text { girls: } 11.15 \pm 0.55 \text { years }\end{array}$ & $1-2$ & $3.40 \pm 0.56$ years & $25 \mathrm{~m}$ & 100 m Freestyle & $\begin{array}{l}\text { Boys: } 284.85 \pm 67.48 \\
\text { Girls: } 322.56 \pm 45.18\end{array}$ \\
\hline Morais et al. (2014b) & $\begin{array}{l}n=14 \text { boys, } 7 \text { high skill: } \\
12.83 \pm 0.37 \text { years, } 7 \text { average skill: } \\
11.83 \pm 0.37 \text { years; } 16 \text { girls, } 8 \text { high } \\
\text { skill: } 11.42 \pm 0.49 \text { years, } 8 \text { average } \\
\text { skill: } 10.83 \pm 0.37 \text { years }\end{array}$ & $1-2$ & $3.40 \pm 0.56$ years & $25 \mathrm{~m}$ & 100 m Freestyle & $\begin{array}{l}\text { Boys (high skill: } \\
294.40 \pm 40.00 ; \text { average skill: } \\
166.20 \pm 17.50 \text { ) } \\
\text { Girls (high skill: } \\
334.30 \pm 39.50 ; \\
\text { average skill: } \\
229.10 \pm 33.90\end{array}$ \\
\hline Morais et al. (2015) & $\begin{array}{l}n=15 \text { boys: } 12.30 \pm 0.60 \text { years; } 18 \\
\text { girls: } 11.70 \pm 0.90 \text { years }\end{array}$ & $1-2$ & $3.18 \pm 0.52$ years & $25 \mathrm{~m}$ & 100 m Freestyle & $\begin{array}{l}\text { Boys: } 227.90 \pm 69.80 \\
\text { Girls: } 291.10 \pm 66.20\end{array}$ \\
\hline Morais et al. (2016) & $\begin{array}{l}n=49 \text { boys: } 12.50 \pm 0.76 \text { years; } 51 \\
\text { girls: } 12.20 \pm 0.71 \text { years }\end{array}$ & $1-2$ & $3.10 \pm 0.71$ years & $25 \mathrm{~m}$ & 100 m Freestyle & n.a. \\
\hline Morais et al. (2017) & $\begin{array}{l}n=47 \text { boys: } 12.04 \pm 0.81 \text { years; } 47 \\
\text { girls: } 11.22 \pm 0.98 \text { years }\end{array}$ & n.a. & $3.18 \pm 0.62$ years & $25 \mathrm{~m}$ & 100 m Freestyle & $\begin{array}{l}\text { Boys: } 217.70 \pm 69.50 \\
\text { Girls: } 277.70 \pm 68.70\end{array}$ \\
\hline Morais et al. (2020a) & $\begin{array}{l}n=22 \text { boys: } 12.79 \pm 0.71 \text { years; } 32 \\
\text { girls: } 11.78 \pm 0.85 \text { years }\end{array}$ & $1-2$ & n.a. & $25 \mathrm{~m}$ & 100 m Freestyle & $\begin{array}{l}\text { Boys: } 297.58 \pm 87.72 \\
\text { Girls: } 330.35 \pm 79.80\end{array}$ \\
\hline Morais et al. (2020b) & $\begin{array}{l}n=14 \text { boys: } 12.70 \pm 0.63 \text { years; } 16 \\
\text { girls: } 11.72 \pm 0.71 \text { years }\end{array}$ & $1-2$ & n.a. & $25 \mathrm{~m}$ & 100 m Freestyle & $\begin{array}{l}\text { Boys: } 234.86 \pm 69.76 \\
\text { Girls: } 288.75 \pm 67.01\end{array}$ \\
\hline Moreira et al. (2014) & $\begin{array}{l}n=12 \text { boys: } 12.80 \pm 0.90 \text { years; } 13 \\
\text { girls: } 12.00 \pm 0.90 \text { years }\end{array}$ & $1-2$ & $3.18 \pm 0.52$ years & n.a. & 25 m Freestyle trial & n.a. \\
\hline Nevill et al. (2020) & $\begin{array}{l}n=n=39 \text { boys: } 11.50 \pm 1.30 \\
\text { years; } n=20 \text { girls: } 12.10 \pm 1.00 \\
\text { years; } n=13.00 \pm 1.00 \text { years }\end{array}$ & $\begin{array}{l}2.33 \pm 1.10 \\
0.04 \pm 1.00 \\
0.82 \pm 0.96 \text { maturity } \\
\text { offset years }\end{array}$ & n.a. & n.a. & $\begin{array}{l}100 \text { m Breaststroke } \\
\text { and Backstroke }\end{array}$ & n.a. \\
\hline Ozeker et al. (2020) & $\begin{array}{l}n=15 \text { girls: } 11.18 \pm 0.80 \text { years; } n= \\
15 \text { girls: } 11.16 \pm 0.83 \text { years }\end{array}$ & n.a. & At least 3 years & $50 \mathrm{~m}$ & $\begin{array}{l}50 \mathrm{~m} \text { and } 400 \mathrm{~m} \\
\text { Freestyle }\end{array}$ & n.a. \\
\hline Poujade et al. (2003) & $\begin{array}{l}n=3 \text { girls and } 8 \text { boys: } 12.40 \pm 0.50 \\
\text { years }\end{array}$ & n.a. & $4-5$ years & $50 \mathrm{~m}$ & 400 m Freestyle & n.a. \\
\hline Poujade et al. (2002) & $\begin{array}{l}n=3 \text { girls and } 8 \text { boys: } 12.40 \pm 0.50 \\
\text { years }\end{array}$ & n.a. & $5-6$ years & n.a. & 400 m Freestyle & n.a. \\
\hline Saavedra et al. (2013) & $n=67$ girls: $11.51 \pm 0.55$ years & n.a. & n.a. & n.a. & $\begin{array}{l}\text { Best score } \\
\text { according to the } \\
\text { LEN table of } \\
\text { competitive } \\
\text { performance level }\end{array}$ & n.a. \\
\hline
\end{tabular}


TABLE 2 | Continued

\begin{tabular}{|c|c|c|c|c|c|c|}
\hline Source & Sample & $\begin{array}{l}\text { Tanner } \\
\text { stage }\end{array}$ & $\begin{array}{l}\text { Years of } \\
\text { experience }\end{array}$ & $\begin{array}{l}\text { Pool } \\
\text { length }\end{array}$ & Race/trial event & $\begin{array}{l}\text { FINA } \\
\text { points }\end{array}$ \\
\hline Saavedra et al. (2010) & $n=67$ girls: $11.50 \pm 0.60$ years & $2.99 \pm 1.19$ & n.a. & $25 \mathrm{~m}$ & $\begin{array}{l}\text { Fastest of three } \\
\text { competitive events } \\
\text { swum in one of the } \\
\text { four strokesat any of } \\
\text { four different race } \\
\text { distances (i.e., } \\
100 \mathrm{~m}, 200 \mathrm{~m}, \\
400 \mathrm{~m} \text {, and } 800 \mathrm{~m} \text { ) }\end{array}$ & n.a. \\
\hline $\begin{array}{l}\text { Sammoud et al. } \\
\text { (2018) }\end{array}$ & $\begin{array}{l}n=39 \text { boys: } 11.50 \pm 1.30 \text { years; } 20 \\
\text { girls: } 12.00 \pm 1.00 \text { years }\end{array}$ & $\begin{array}{l}\text { Boys: }-2.30 \pm 1.10 \\
\text { girls: } 0.04 \pm 1.00 \\
\text { maturity offset years }\end{array}$ & n.a. & $25 \mathrm{~m}$ & $100 \mathrm{~m}$ Breaststroke & n.a. \\
\hline $\begin{array}{l}\text { Sammoud et al. } \\
\text { (2019) }\end{array}$ & $\begin{array}{l}(n=26 \text { boys) two groups: } \\
10.30 \pm 0.40 \text { and } 10.50 \pm 0.40 \text { years }\end{array}$ & $\begin{array}{l}-3.10 \pm 0.30 \text { and } \\
-2.80 \pm 0.30 \text { years } \\
\text { until peak height } \\
\text { velocity }\end{array}$ & $2.00 \pm 1.60$ years & $50 m$ & $\begin{array}{l}15 \mathrm{~m}, 25 \mathrm{~m} \text {, and } \\
50 \mathrm{~m} \text { Freestyle trial }\end{array}$ & n.a. \\
\hline Seffrin et al. (2021) & $\begin{array}{l}n=16 \text { boys: } 11.50 \pm 0.52 \text { years; } 6 \\
\text { girls: } 11.67 \pm 0.52 \text { years }\end{array}$ & n.a. & n.a. & n.a. & $\begin{array}{l}100 \mathrm{~m} \text { and } 400 \mathrm{~m} \\
\text { Freestyle }\end{array}$ & n.a. \\
\hline Silva et al. (2012) & $\begin{array}{l}\text { ( } n=36 \text { boys: } 12.42 \pm 0.08 \text { years; } \\
\text { and } 24 \text { girls: } 11.08 \pm 0.08 \text { years) }\end{array}$ & $\begin{array}{l}\text { Boys: } 2-3 \\
\text { Girls: } 2-3\end{array}$ & $\begin{array}{l}3.75 \pm 0.87 \text { and } \\
3.38 \pm 0.77 \text { years }\end{array}$ & n.a. & $\begin{array}{l}25 \mathrm{~m} \text { Backstroke } \\
\text { trial }\end{array}$ & n.a. \\
\hline Silva et al. (2013) & $\begin{array}{l}\text { Pubertal: } \mathrm{n}=36 \text { boys: } 12.42 \pm 0.08 \\
\text { years; } 24 \text { girls: } 11.08 \pm 0.08 \text { years } \\
\text { Post-pubertal: } n=20 \text { boys: } \\
12.65 \pm 0.11 \text { years; } 34 \text { girls: } \\
11.71 \pm 0.08 \text { years }\end{array}$ & $\begin{array}{l}\text { Pubertal: 1-2 } \\
\text { Post-pubertal: 3-5 }\end{array}$ & $\begin{array}{l}\text { Pubertal boys: } \\
3.75 \pm 0.87 \text { years; } \\
\text { girls: } \\
3.38 \pm 0.77 \text { years } \\
\text { Post-pubertal } \\
\text { boys: } 3.75 \pm 1.25 \\
\text { years; girls: } \\
3.35 \pm 1.07 \text { years }\end{array}$ & n.a. & $25 \mathrm{~m}$ Freestyle trial & n.a. \\
\hline Staub et al. (2020a) & $n=842$ boys and 863 girls: 11 years & n.a. & n.a. & & $\begin{array}{l}50 \mathrm{~m}, 100 \mathrm{~m}, 200 \mathrm{~m} \text {, } \\
\text { and } 400 \mathrm{~m} \text { Freestyle; } \\
50 \mathrm{~m}, 100 \mathrm{~m} \text {, and } \\
200 \mathrm{~m} \text { for both } \\
\text { Breaststroke and } \\
\text { Backstroke; } 50 \mathrm{~m} \text {, } \\
\text { and } 100 \mathrm{~m} \text { Butterfly; } \\
200 \mathrm{~m} \text { Individual } \\
\text { Medley }\end{array}$ & $\begin{array}{l}\text { Relationships between } \\
\text { success at age } 18 \text { (1-1000 } \\
\text { FINA points), to within-sport } \\
\text { specialization and age of entry }\end{array}$ \\
\hline Tijani et al. (2019) & $\begin{array}{l}n=22 \text { boys and } 18 \\
\text { girls } 12.30 \pm 0.56 \text { years }\end{array}$ & n.a. & $7.10 \pm 0.50$ years & $25 \mathrm{~m}$ & 50 m Freestyle & n.a. \\
\hline Tsalis et al. (2012) & $n=8$ girls: $10.40 \pm 0.60$ years & n.a. & n.a. & $50 \mathrm{~m}$ & $\begin{array}{l}50 \mathrm{~m}, 100 \mathrm{~m}, 200 \mathrm{~m}, \\
\text { and } 400 \mathrm{~m} \text { Freestyle }\end{array}$ & n.a. \\
\hline $\begin{array}{l}\text { Zarzeczny et al. } \\
\text { (2013) }\end{array}$ & $n=24$ boys: $12.20 \pm 0.10$ years & n.a. & n.a. & $25 \mathrm{~m}$ & $\begin{array}{l}50 \mathrm{~m}, \text { and } 400 \mathrm{~m} \\
\text { Freestyle and } \\
\text { Breaststroke }\end{array}$ & n.a. \\
\hline
\end{tabular}

n.a., not applicable (i.e., not reported).

in the 100-m breaststroke and backstroke events (Nevill et al., 2020). This review only includes data related to breaststroke and backstroke from this article (Nevill et al., 2020) because only these strokes met the inclusion criteria (i.e., under 13 years of average age). Nonetheless, the authors agreed that such characteristics were common in the whole sample (over 13 years 


\begin{tabular}{|c|c|c|c|c|c|c|c|}
\hline \multirow[b]{2}{*}{ Source } & \multirow[b]{2}{*}{ Purpose } & \multirow[b]{2}{*}{ Design } & \multirow[b]{2}{*}{ Anthropometrics } & \multirow[b]{2}{*}{ Biomechanics } & \multirow[b]{2}{*}{$\begin{array}{l}\text { Energetics/ } \\
\text { Efficiency }\end{array}$} & \multicolumn{2}{|c|}{ Performance } \\
\hline & & & & & & Initial & Final \\
\hline $\begin{array}{l}\text { Abbes et al. } \\
\text { (2018) }\end{array}$ & $\begin{array}{l}\text { To investigate whether tethered swimming before } \\
\text { a } 50 \mathrm{~m} \text { freestyle swimming sprint could be an } \\
\text { effective post-activation potentiation method to } \\
\text { improve performance }\end{array}$ & Longitudinal & $\mathrm{BM}, \mathrm{H}$ & CMJ, SL & $\mathrm{RPE}, \mathrm{BI}$ & \multicolumn{2}{|c|}{$\begin{array}{l}50 \text { Free CG: } 32.48 \pm 3.35 \mathrm{~s} \\
50 \text { Free EG: } 32.68 \pm 3.68 \mathrm{~s}\end{array}$} \\
\hline $\begin{array}{l}\text { Abbes et al. } \\
(2020)\end{array}$ & $\begin{array}{l}\text { To investigate performance, biomechanical, } \\
\text { physiological, and psychophysiological effects of a } \\
\text { simple and easily organized } \\
\text { post-activationpotentiation re-warm-up performed } \\
\text { before a } 50 \mathrm{~m} \text { freestyle swimming sprint }\end{array}$ & Longitudinal & $\mathrm{BM}, \mathrm{H}$ & SF, SL & $\mathrm{RPE}, \mathrm{Bl}, \mathrm{HR}$ & \multicolumn{2}{|c|}{$\begin{array}{c}50 \text { Free Push-ups group: } 32.62 \pm 2.81 \mathrm{~s} \\
50 \text { Free Squat jump group: } 32.42 \pm 2.32 \mathrm{~s} \\
50 \text { Free Burpees group: } 32.46 \pm 2.26 \mathrm{~s} \\
50 \text { Free CG: } 32.84 \pm 2.53 \mathrm{~s}\end{array}$} \\
\hline $\begin{array}{l}\text { Abbott et al. } \\
(2021)\end{array}$ & $\begin{array}{l}\text { To examine the longitudinal relationships between } \\
\text { maturity status, technical skill indices, and } \\
\text { performance in male youth competitiveswimmers. } \\
\text { To determine whether individualdifferences in } \\
\text { maturation influenced relationships between } \\
\text { technicalskill level and swim performance. }\end{array}$ & $\begin{array}{l}\text { Longitudinal ( } 4 \\
\text { months) }\end{array}$ & $\mathrm{BM}, \mathrm{H}$ & $\mathrm{v}$ & $\mathrm{SI}, \eta_{\mathrm{F}}$ & $\begin{array}{l}200 \text { Free }\left(10 \text { years): } 1.08 \pm 0.08 \mathrm{~m} \cdot \mathrm{s}^{-1}\right. \\
200 \text { Free (11 years): } 1.16 \pm 0.08 \mathrm{~m} \cdot \mathrm{s}^{-1} \\
200 \text { Free (12 years): } 1.21 \pm 0.09 \mathrm{~m} \cdot \mathrm{s}^{-1} \\
200 \text { Free (13 years): } 1.23 \pm 0.12 \mathrm{~m} \cdot \mathrm{s}^{-1}\end{array}$ & $\begin{array}{l}200 \text { Free (11 years): } \\
1.20 \pm 0.12 \mathrm{~m} \cdot \mathrm{s}^{-1} 200 \text { Free }(12 \\
\text { years): } 1.26 \pm 0.08 \mathrm{~m} \cdot \mathrm{s}^{-1} 200 \text { Free } \\
\text { (13 years): } 1.28 \pm 0.07 \mathrm{~m} \cdot \mathrm{s}^{-1} 200 \\
\text { Free (14 years): } 1.23 \pm 0.12 \mathrm{~m} \cdot \mathrm{s}^{-1}\end{array}$ \\
\hline $\begin{array}{l}\text { Alshdokhi } \\
\text { et al. (2020) }\end{array}$ & $\begin{array}{l}\text { To quantify and compare the transfer of dryland } \\
\text { strength gains to adolescent backstroke and } \\
\text { freestyle swimming performance }\end{array}$ & $\begin{array}{l}\text { Longitudinal (8 } \\
\text { weeks) }\end{array}$ & $\mathrm{BM}, \mathrm{H}, \mathrm{RH}$ & $\begin{array}{l}\mathrm{SF}, \mathrm{VJ}, \mathrm{BJ}, \mathrm{PC}, \\
L F_{\text {ext }}, \mathrm{RF} \mathrm{Fext}_{\mathrm{ext}}, \mathrm{LF} \text { int, } \\
\mathrm{RF} \text { int, } \mathrm{BE}\end{array}$ & $\mathrm{HR}, \mathrm{RPE}$ & $\begin{array}{l}50 \text { Free CG: } 43.93 \pm 7.11 \mathrm{~s} \\
50 \text { Free EG: } 44.23 \pm 10.27 \mathrm{~s} \\
50 \text { Back CG: } 49.58 \pm 6.31 \mathrm{~s} \\
50 \text { Back EG: } 49.18 \pm 7.00 \mathrm{~s} \\
100 \text { Free CG: } 104.60 \pm 12.35 \mathrm{~s} \\
100 \text { Free EG: } 102.58 \pm 21.72 \mathrm{~s} \\
100 \text { Back CG: } 119.48 \pm 18.69 \mathrm{~s} \\
100 \text { Back EG: } 113.81 \pm 22.02 \mathrm{~s}\end{array}$ & $\begin{array}{c}\text { 50 Free CG: } 42.78 \pm 7.13 \text { s } 50 \text { Free } \\
\text { EG: } 42.19 \pm 10.23 \text { s } 50 \text { Back CG: } \\
47.87 \pm 6.88 \text { s } 50 \text { Back EG: } \\
47.08 \pm 7.41 \text { s } 100 \text { Free CG: } \\
102.98 \pm 12.33 \text { s } 100 \text { Free EG: } \\
99.08 \pm 22.32 \text { s } 100 \text { Back CG: } \\
118.01 \pm 18.89 \text { s } 100 \text { Back EG: } \\
112.01 \pm 21.77 \mathrm{~s}\end{array}$ \\
\hline $\begin{array}{l}\text { Amaro et al. } \\
(2017)\end{array}$ & $\begin{array}{l}\text { To analyze the effects of a period of swim training } \\
\text { alone (CG), a dryland SC program based on } \\
\text { sets/repetitions (EG1), plus swim training alone or } \\
\text { a dryland SandC program that focused on } \\
\text { explosiveness plus swim training alone (EG2) }\end{array}$ & $\begin{array}{l}\text { Longitudinal (10 } \\
\text { weeks) }\end{array}$ & $\mathrm{BM}, \mathrm{H}$ & MF, MMI, VJ, BT & n.a. & $\begin{array}{l}50 \text { Free CG: } 33.76 \pm 3.14 \mathrm{~s} \\
50 \text { Free EG1: } 33.92 \pm 1.47 \mathrm{~s} \\
50 \text { Free EG2: } 33.43 \pm 2.83 \mathrm{~s}\end{array}$ & $\begin{array}{c}\text { 50 Free CG: } 33.64 \pm 3.04 \mathrm{~s} 50 \text { Free } \\
\text { EG1: } 34.02 \pm 1.61 \mathrm{~s} 50 \text { Free EG2: } \\
31.65 \pm 2.53 \mathrm{~s}\end{array}$ \\
\hline $\begin{array}{l}\text { Barbosa et al. } \\
\text { (2010) }\end{array}$ & $\begin{array}{l}\text { To develop a model for young swimmers' } \\
\text { performance based on biomechanical and } \\
\text { energetic parameters }\end{array}$ & Cross-sectional & $\mathrm{BM}, \mathrm{H}, \mathrm{FM}$ & $S L, S F, v$ & $\mathrm{CV}, \mathrm{SI}, \eta_{\mathrm{F}}$ & 200 Free: 156.80 & $\pm 17.30 \mathrm{~s}$ \\
\hline $\begin{array}{l}\text { Barbosa et al. } \\
\text { (2014) }\end{array}$ & $\begin{array}{l}\text { To develop a classification system for young } \\
\text { talented swimmers based on kinematical, } \\
\text { hydrodynamic, and anthropometrical } \\
\text { characteristics }\end{array}$ & Cross-sectional & FSA & $v, d v, d v / v, C_{D a}$ & n.a. & 100 Free: 71.30 & $\pm 6.12 \mathrm{~s}$ \\
\hline $\begin{array}{l}\text { Barbosa et al. } \\
\text { (2015) }\end{array}$ & $\begin{array}{l}\text { To compare swimming power output between } \\
\text { boys and girls, and model the relationship } \\
\text { between swimming power output and sprinting } \\
\text { performance }\end{array}$ & Cross-sectional & $\mathrm{BM}, \mathrm{H}, \mathrm{AS}, \mathrm{FSA}$ & $\begin{array}{l}\text { SF, SL, SL/AS, v, } \\
d v, d v / v, D_{a}, C_{D A} \\
P_{d}, P_{k}, P_{\text {ext }}\end{array}$ & $S I, \eta_{F}$ & $\begin{array}{l}\text { Boys } 100 \text { Free: } 1.44 \\
\text { Girls } 100 \text { Free: } 1.30=\end{array}$ & $\begin{array}{l} \pm 0.16 \mathrm{~m} \cdot \mathrm{s}^{-1} \\
\pm 0.12 \mathrm{~m} \cdot \mathrm{s}^{-1}\end{array}$ \\
\hline $\begin{array}{l}\text { Barbosa et al. } \\
\text { (2019) }\end{array}$ & $\begin{array}{l}\text { To compare the anthropometrics, biomechanics } \\
\text { and energetics in young swimmers of different } \\
\text { competitive levels }\end{array}$ & Cross-sectional & $\mathrm{BM}, \mathrm{H}, \mathrm{AS}, \mathrm{FSA}$ & $\begin{array}{l}\text { SF, SL, SL/AS, v, } \\
D_{a}, C_{D A}, P_{d}, P_{k}, \\
P_{\text {ext }}, E_{\text {tot }}, F_{r}, V_{h}, R_{e}\end{array}$ & $\mathrm{SI}, \eta_{\mathrm{F}}, \mathrm{dv}$ & $\begin{array}{l}100 \text { Free Tier } 1: 1.75 \\
100 \text { Free Tier 2: } 1.53 \\
100 \text { Free Tier 3: } 1.38\end{array}$ & $\begin{array}{l} \pm 0.07 \mathrm{~m} \cdot \mathrm{s}^{-1} \\
\pm 0.11 \mathrm{~m} \cdot \mathrm{s}^{-1} \\
\pm 0.13 \mathrm{~m} \cdot \mathrm{s}^{-1}\end{array}$ \\
\hline $\begin{array}{l}\text { Bielec and } \\
\text { Jurak (2019) }\end{array}$ & $\begin{array}{l}\text { To describe the anthropometric characteristics of } \\
\text { prepubescent swimmers and to determine the } \\
\text { contribution of chosen anthropometric factors to } \\
\text { sports achievements }\end{array}$ & Cross-sectional & $\begin{array}{l}\mathrm{H}, \mathrm{HW}, \mathrm{HL}, \mathrm{AS}, \\
\mathrm{BM}, \mathrm{BMI}, \mathrm{BF}\end{array}$ & v & n.a. & n.a. & \\
\hline
\end{tabular}


Performance

\begin{tabular}{|c|c|c|c|c|c|c|}
\hline \multirow[b]{2}{*}{ Source } & \multirow[b]{2}{*}{ Purpose } & \multirow[b]{2}{*}{ Design } & \multirow[b]{2}{*}{ Anthropometrics } & \multirow[b]{2}{*}{ Biomechanics } & \multirow[b]{2}{*}{$\begin{array}{l}\text { Energetics/ } \\
\text { Efficiency }\end{array}$} & Performance \\
\hline & & & & & & Initial \\
\hline $\begin{array}{l}\text { Costa et al. } \\
\text { (2011) }\end{array}$ & $\begin{array}{l}\text { To track and analyze freestyle performance during } \\
\text { elite-standard male swimmers' careers, from } 12 \text { to } \\
18 \text { years of age }\end{array}$ & $\begin{array}{l}\text { Longitudinal } \\
\text { (12 to } 18 \\
\text { years-old) }\end{array}$ & n.a. & n.a. & n.a. & $\begin{array}{l}50 \text { Free: } \Delta=5.85 \pm 2.66 \% \\
100 \text { Free: } \Delta=4.89 \pm 2.70 \% \\
\text { 200 Free } \Delta=5.54 \pm 2.23 \% \\
\text { 400 Free: } \Delta=5.47 \pm 2.23 \% \\
800 \text { Free: } \Delta=5.74 \pm 3.24 \% \\
\text { 1500 Free: } \Delta=5.34 \pm 2.69 \%\end{array}$ \\
\hline $\begin{array}{l}\text { de Mello Vitor } \\
\text { and Böhme } \\
\text { (2010) }\end{array}$ & $\begin{array}{l}\text { To assess the relationship among anthropometric } \\
\text { variables, specific physical conditioning, } \\
\text { swimming techniques and } 100 \mathrm{~m} \text { Freestyle } \\
\text { performance }\end{array}$ & Cross-sectional & $\begin{array}{l}\text { BM, H, AS, HL, } \\
\text { HW, FL, FW, Biacr } \\
\text { B, Bililiac B, AS/H, } \\
\text { Biacr B/Biliac B, } \\
\text { TS, SS, BF }\end{array}$ & SF, SL, SI & AnP, CV & 100 Free: $1.46 \pm 0.07 \mathrm{~m} \cdot \mathrm{s}^{-1}$ \\
\hline $\begin{array}{l}\text { Denadai et al. } \\
\text { (2000) }\end{array}$ & $\begin{array}{l}\text { To verify whether critical speed can be used as a } \\
\text { non-invasive method for the determination of } \\
\text { speed at a blood lactate concentration of } 4 \\
\text { mmol. } \cdot^{-1}\end{array}$ & Cross-sectional & $\mathrm{BM}, \mathrm{H}$ & $v$ & $\mathrm{CV}, \mathrm{BI}, \mathrm{V} 4$ & $\begin{array}{l}\text { Beginner CV: } 0.78 \pm 0.25 \mathrm{~m} \cdot \mathrm{s}^{-1} \\
\text { Beginner V4: } 0.82 \pm 0.09 \mathrm{~m} \cdot \mathrm{s}^{-1} \\
\text { Trained CV: } 1.08 \pm 0.4 \mathrm{~m} \cdot \mathrm{s}^{-1} \\
\text { Trained V4: } 1.19 \pm 0.11 \mathrm{~m} \cdot \mathrm{s}^{-1}\end{array}$ \\
\hline $\begin{array}{l}\text { Duché et al. } \\
\text { (1993) }\end{array}$ & $\begin{array}{l}\text { To determine the influence of anthropometric and } \\
\text { bio-energetic parameters on } \\
\text { swimming performance }\end{array}$ & Cross-sectional & $\begin{array}{l}\text { H, SH, BM, BF, } \\
\text { Biacr B, Biiliac B, } \\
\text { TSA, BA, ULL, AL, } \\
\text { ForL }\end{array}$ & $v$ & $\begin{array}{l}\mathrm{VO}_{2 \max }, \mathrm{AnP} \\
\mathrm{MP}_{30}\end{array}$ & $\begin{array}{l}50 \text { Free: } 40.60 \pm 7.20 \mathrm{~s} \\
100 \text { Free: } 85.60 \pm 14.70 \mathrm{~s} \\
200 \text { Free: } 187.70 \pm 30.60 \mathrm{~s} \\
\text { 400 Free: } 399.00 \pm 78.50 \mathrm{~s}\end{array}$ \\
\hline $\begin{array}{l}\text { Ferraz et al. } \\
(2020)\end{array}$ & $\begin{array}{l}\text { To verifyassociations between the } \\
\text { anthropometriccharacteristics of young swimmers } \\
\text { ofdifferent genders and different competitive levels } \\
\text { with sports performance in the } 50 \mathrm{~m} \text { and } 400 \mathrm{~m} \\
\text { freestyle races at different levels. }\end{array}$ & Cross-sectional & $\begin{array}{l}\mathrm{BM}, \mathrm{H}, \mathrm{BMl}, \mathrm{AS} \\
\mathrm{AS} / \mathrm{H}\end{array}$ & $S F, S L$ & $\mathrm{SI}$ & $\begin{array}{c}\text { Boys (U12) } 50 \mathrm{~m} \text { Free: } 33.20 \pm 1.98 \mathrm{~s} \\
\text { Boys (U12) } 400 \mathrm{~m} \text { Free: } 326.48 \pm 16.94 \mathrm{~s} \\
\text { Girls (U13) } 50 \mathrm{~m} \text { Free: } 34.48 \pm 2.34 \mathrm{~s} \\
\text { Girls (U13) } 400 \mathrm{~m} \text { Free: } 330.75 \pm 25.92 \mathrm{~s} \\
\text { Girls (U12) } 50 \mathrm{~m} \text { Free: } 36.52 \pm 1.85 \mathrm{~s} \\
\text { Girls (U12) } 400 \mathrm{~m} \text { Free: } 364.18 \pm 26.36 \mathrm{~s}\end{array}$ \\
\hline $\begin{array}{l}\text { Ferreira et al. } \\
\text { (2019) }\end{array}$ & $\begin{array}{l}\text { To examine the physiological and biomechanical } \\
\text { responses related to the } 400 \mathrm{~m} \\
\text { swimming performance }\end{array}$ & $\begin{array}{l}\text { Longitudinal (11 } \\
\text { weeks) }\end{array}$ & $\mathrm{BM}, \mathrm{H}$ & SF, SL & $\mathrm{SI}, \mathrm{HR}, \mathrm{BI}, \mathrm{Bg}$ & 400 Free: $444.40 \pm 76.95 \mathrm{~s} \quad 400$ Free: $408.95 \pm 61.40 \mathrm{~s}$ \\
\hline $\begin{array}{l}\text { Ferreira et al. } \\
\text { (2021) }\end{array}$ & $\begin{array}{l}\text { To describe the evolution of middle-distance } \\
\text { swimming performancealong with physiological } \\
\text { and biomechanical changes in young swimmers } \\
\text { during a trainingseason including three } \\
\text { macrocycles. }\end{array}$ & $\begin{array}{l}\text { Longitudinal (45 } \\
\text { weeks) }\end{array}$ & $\mathrm{BM}, \mathrm{H}, \mathrm{BMI}$ & $S F, S L$ & $\begin{array}{l}\mathrm{SI}, \mathrm{HR}, \mathrm{Bl}, \mathrm{Bg}, \\
\mathrm{RPE}\end{array}$ & 400 Free: $432.37 \pm 71.78 \mathrm{~s}$ \\
\hline $\begin{array}{l}\text { Figueiredo } \\
\text { et al. (2016) }\end{array}$ & $\begin{array}{l}\text { To evaluate the determinants of front crawl } \\
\text { swimming sprint performance }\end{array}$ & Cross-sectional & $\begin{array}{l}\mathrm{BM}, \mathrm{H}, \mathrm{AS}, \mathrm{HL} \\
\mathrm{HW}, \mathrm{FL}, \mathrm{FW}\end{array}$ & $\begin{array}{l}\text { SF, SL, SL/AS, dv, } \\
\text { IdC }\end{array}$ & CV, SI, $\eta_{F}$ & $\begin{array}{l}25 \text { Free Cluster 1: } 1.52 \pm 0.16 \mathrm{~m} \cdot \mathrm{s}^{-1} \\
25 \text { Free Cluster 2: } 1.47 \pm 0.17 \mathrm{~m} \cdot \mathrm{s}^{-1} \\
25 \text { Free Cluster } 3: 1.40 \pm 0.15 \mathrm{~m} \cdot \mathrm{s}^{-1}\end{array}$ \\
\hline $\begin{array}{l}\text { Garrido et al. } \\
\text { (2010a) }\end{array}$ & $\begin{array}{l}\text { To identify the dryland strength and power tests } \\
\text { that can better associate with sprint swimming } \\
\text { performance }\end{array}$ & Cross-sectional & $\mathrm{BM}, \mathrm{H}$ & $\begin{array}{l}\text { LE, BP, CMJ, BT, } \\
\text { BR }\end{array}$ & n.a. & $\begin{array}{l}25 \text { Free: } 16.12 \pm 0.67 \mathrm{~s} \\
50 \text { Free: } 35.21 \pm 1.98 \mathrm{~s}\end{array}$ \\
\hline $\begin{array}{l}\text { Garrido et al. } \\
\text { (2010b) }\end{array}$ & $\begin{array}{l}\text { To examine the effects of combined dryland } \\
\text { strength and aerobic swimming training for } \\
\text { increasing upper and lower body strength, power } \\
\text { and swimming performance }\end{array}$ & $\begin{array}{l}\text { Longitudinal } \\
\text { (8 weeks) }\end{array}$ & $\mathrm{BM}, \mathrm{H}$ & $\begin{array}{l}\mathrm{D}_{\mathrm{a}}, \mathrm{C}_{\mathrm{Da}}, \mathrm{LE}, \mathrm{BP} \\
\mathrm{CMJ}, \mathrm{BT}, \mathrm{BR}\end{array}$ & n.a. & $\begin{array}{l}\text { EG } 25 \text { Free: } \Delta=6.95 \% \\
\text { EG } 50 \text { Free: } \Delta=4.77 \%\end{array}$ \\
\hline $\begin{array}{l}\text { Geladas et al. } \\
\text { (2005) }\end{array}$ & $\begin{array}{l}\text { To examine the relationship between } \\
\text { anthropometry, some physical capacity traits and } \\
\text { sprint swimming performance }\end{array}$ & Cross-sectional & $\begin{array}{l}\text { BM, BF, H, TUEL, } \\
\text { HL, FL, CC, Biacr } \\
\text { B,Biiliac B, AFlex, } \\
\text { SFlex, }\end{array}$ & $\mathrm{HJ}, \mathrm{HG}$ & n.a. & $\begin{array}{l}\text { Boys } 100 \text { Free: } 65.52 \pm 0.25 \mathrm{~s} \\
\text { Girls } 100 \text { Free: } 68.10 \pm 0.22 \mathrm{~s}\end{array}$ \\
\hline
\end{tabular}


Performance

\begin{tabular}{|c|c|c|c|c|c|c|}
\hline Source & Purpose & Design & Anthropometrics & Biomechanics & $\begin{array}{l}\text { Energetics/ } \\
\text { Efficiency }\end{array}$ & Initial \\
\hline $\begin{array}{l}\text { Hue et al. } \\
\text { (2013) }\end{array}$ & $\begin{array}{l}\text { To investigate the anthropometric and } \\
\text { physiological characteristics of young } \\
\text { Guadeloupian competitive swimmers }\end{array}$ & Cross-sectional & $\mathrm{BM}, \mathrm{BF}, \mathrm{H}, \mathrm{AS}, \mathrm{LL}$ & CMJ, HL, Glide & $\mathrm{eVO}_{2 \max }, \mathrm{MAV}$ & $\begin{array}{l}\text { Boys } 15 \text { Free: } 10.25 \pm 0.33 \mathrm{~s} \\
\text { Boys } 400 \text { Free: } 363.75 \pm 20.16 \mathrm{~s} \\
\text { Girls } 15 \text { Free: } 10.63 \pm 0.21 \mathrm{~s} \\
\text { Girls } 400 \text { Free: } 359.25 \pm 14.86 \mathrm{~s}\end{array}$ \\
\hline $\begin{array}{l}\text { Jürimäe et al. } \\
\text { (2007) }\end{array}$ & $\begin{array}{l}\text { To examine the influence of energy cost } \\
\text { of swimming, anthropometrical, body } \\
\text { composition, and technical parameters on } \\
\text { swimming performance }\end{array}$ & Cross-sectional & $\begin{array}{l}\text { BM, BF, BMI, BMM, } \\
\text { H, FM, FFM, AS, } \\
\text { TBMD, SBMD }\end{array}$ & SF, SL, v & $\begin{array}{l}\mathrm{Sl}, \mathrm{C}_{\mathrm{s}}, \mathrm{VO}_{2} \\
\Delta \mathrm{La}\end{array}$ & 400 Free: $401.50 \pm 53.80 \mathrm{~s}$ \\
\hline $\begin{array}{l}\text { Kjendlie et al. } \\
\text { (2004a) }\end{array}$ & $\begin{array}{l}\text { To investigate the differences in the energy cost at } \\
\text { submaximal velocities in boys, and to study the } \\
\text { differences in the energy cost at different size } \\
\text { scaled submaximal velocities }\end{array}$ & Cross-sectional & BL, BM, BSA & $\mathrm{Bu}, \mathrm{Vol}$ & $\mathrm{C}_{\mathrm{s}}, \mathrm{VO}_{2}$ & $\begin{array}{l}50 \text { Free: } 33.70 \pm 2.90 \mathrm{~s} \\
100 \text { Free: } 75.10 \pm 5.50 \mathrm{~s}\end{array}$ \\
\hline $\begin{array}{l}\text { Kjendlie and } \\
\text { Stallman } \\
\text { (2008) }\end{array}$ & $\begin{array}{l}\text { To compare drag in swimming children, quantify } \\
\text { technique using the technique drag index, anduse } \\
\text { the Froude number to study whether children } \\
\text { reach hull speed at maximal swim speed }\end{array}$ & Cross-sectional & $\mathrm{BL}, \mathrm{BM}, \mathrm{BSA}, \mathrm{H}$ & $\begin{array}{l}R_{e}, F_{r}, D_{a}, C_{D a}, D_{p}, \\
C_{D p}, T D I, v\end{array}$ & n.a. & 25 Free: $1.42 \pm 0.12 \mathrm{~m} \cdot \mathrm{s}^{-1}$ \\
\hline $\begin{array}{l}\text { Lätt et al. } \\
\text { (2009a) }\end{array}$ & $\begin{array}{l}\text { To examine the development of specific physical, } \\
\text { physiological, and biomechanical parameters } \\
\text { during swimmers' maturing and the influence of } \\
\text { such parameters on swimming performance }\end{array}$ & $\begin{array}{l}\text { Longitudinal } \\
\text { (2years) }\end{array}$ & $\begin{array}{l}\text { BM, BMI, BF, H, } \\
\text { AS, FM, BMM, } \\
\text { FFM, TBMD, SBMD }\end{array}$ & $\mathrm{v}, \mathrm{SF}, \mathrm{SL}$ & $\begin{array}{l}\mathrm{Sl}, \mathrm{C}_{\mathrm{s}} \\
\mathrm{VO}_{2}, \Delta \mathrm{La}\end{array}$ & 400 Free: $373.30 \pm 53.50 \mathrm{~s}$ \\
\hline $\begin{array}{l}\text { Lätt et al. } \\
\text { (2009b) }\end{array}$ & $\begin{array}{l}\text { To examine the development of anthropometrical, } \\
\text { physiological, and biomechanical parameters } \\
\text { during swimmers' maturing and the influence of } \\
\text { such parameters on swimming performance }\end{array}$ & $\begin{array}{l}\text { Longitudinal } \\
\text { (2years) }\end{array}$ & $\begin{array}{l}\text { BM, BMI, BF, H, } \\
\text { AS, FM, BMM, } \\
\text { FFM, TBMD, SBMD }\end{array}$ & v, SF, SL & $\begin{array}{l}\mathrm{SI}, \mathrm{C}_{\mathrm{s}}, \\
\mathrm{VO}_{2}, \Delta \mathrm{La}\end{array}$ & 400 Free: $373.90 \pm 39.20 \mathrm{~s}$ \\
\hline $\begin{array}{l}\text { Majid et al. } \\
\text { (2019) }\end{array}$ & $\begin{array}{l}\text { To recognize the effect of special exercises in the } \\
\text { development of the rapid strength of the muscles } \\
\text { of the legs and arms and the completion of the } \\
50 \mathrm{~m} \text { breaststroke }\end{array}$ & Longitudinal & $\mathrm{BM}, \mathrm{H}$ & $\mathrm{AE}, \mathrm{KFE}$ & n.a. & 50 Breast: $49.84 \pm 5.51 \mathrm{~s}$ \\
\hline $\begin{array}{l}\text { Marinho et al. } \\
\text { (2011) }\end{array}$ & $\begin{array}{l}\text { To determine and analyze the anaerobic critical } \\
\text { velocity comparing it with short distances } \\
\text { performances in the four swimming techniques }\end{array}$ & Cross-sectional & $\mathrm{BM}, \mathrm{H}$ & n.a. & AnCV & $\begin{array}{c}50 \mathrm{~m} \text { Free: } 1.45 \pm 0.18 \mathrm{~m} \cdot \mathrm{s}^{-1} \\
100 \mathrm{~m} \text { Free: } 1.39 \pm 0.17 \mathrm{~m} \cdot \mathrm{s}^{-1} \\
200 \mathrm{~m} \text { Free: } 1.29 \pm 0.14 \mathrm{~m} \cdot \mathrm{s}^{-1} \\
50 \mathrm{~m} \text { Fly: } 1.36 \pm 0.18 \mathrm{~m} \cdot \mathrm{s}^{-1} \\
100 \mathrm{~m} \text { Fly: } 1.23 \pm 0.14 \mathrm{~m} \cdot \mathrm{s}^{-1} \\
200 \mathrm{~m} \text { Fly: } 1.08 \pm 0.11 \mathrm{~m} \cdot \mathrm{s}^{-1} \\
50 \mathrm{~m} \text { Back: } 1.21 \pm 0.09 \mathrm{~m} \cdot \mathrm{s}^{-1} \\
100 \mathrm{~m} \text { Back: } 1.17 \pm 0.09 \mathrm{~m} \cdot \mathrm{s}^{-1} \\
200 \mathrm{~m} \text { Back: } 1.13 \pm 0.09 \mathrm{~m} \cdot \mathrm{s}^{-1} \\
50 \mathrm{~m} \text { Breast: } 1.09 \pm 0.16 \mathrm{~m} \cdot \mathrm{s}^{-1} \\
100 \mathrm{~m} \text { Breast: } 1.04 \pm 0.13 \mathrm{~m} \cdot \mathrm{s}^{-1} \\
200 \mathrm{~m} \text { Breast: } 0.93 \pm 0.11 \mathrm{~m} \cdot \mathrm{s}^{-1}\end{array}$ \\
\hline $\begin{array}{l}\text { Marinho et al. } \\
\text { (2020) }\end{array}$ & $\begin{array}{l}\text { To understand the relationship between the } \\
\text { coaches' demographics (academic degree, } \\
\text { coaching level, training experience) in the applied } \\
\text { training content and the swimmers' technical } \\
\text { ability and performance. }\end{array}$ & Cross-sectional & $\mathrm{BM}, \mathrm{H}, \mathrm{AS}$ & $\begin{array}{l}\mathrm{v}, \mathrm{dv}, \mathrm{SL}, \mathrm{R}_{\mathrm{e}}, \mathrm{F}_{\mathrm{r}}, \\
\mathrm{C}_{\mathrm{Da}}\end{array}$ & $\mathrm{SI}, \eta_{\mathrm{F}}$ & $\begin{array}{c}100 \mathrm{~m} \text { Free (Acad_level_1): } 75.51 \pm 10.02 \mathrm{~s} \\
100 \mathrm{~m} \text { Free (Acad_level_2): } 74.55 \pm 9.56 \mathrm{~s} \\
100 \mathrm{~m} \text { Free (Acad_level_3): } 73.62 \pm 7.64 \mathrm{~s} \\
100 \mathrm{~m} \text { Free (Coach_level_1): } 76.79 \pm 11.27 \mathrm{~s} \\
100 \mathrm{~m} \text { Free (Coach_level_2): } 75.06 \pm 9.31 \mathrm{~s} \\
100 \mathrm{~m} \text { Free (Coach_level_3): } 73.65 \pm 8.43 \mathrm{~s} \\
100 \mathrm{~m} \text { Free (Exp__ 5): } 75.44 \pm 9.57 \mathrm{~s} \\
100 \mathrm{~m} \text { Free (Exp_> 5): } 74.60 \pm 9.54 \mathrm{~s}\end{array}$ \\
\hline
\end{tabular}




\begin{tabular}{|c|c|c|c|c|c|c|c|}
\hline \multirow[b]{2}{*}{ Source } & \multirow[b]{2}{*}{ Purpose } & \multirow[b]{2}{*}{ Design } & \multirow[b]{2}{*}{ Anthropometrics } & \multirow[b]{2}{*}{ Biomechanics } & \multirow[b]{2}{*}{$\begin{array}{l}\text { Energetics/ } \\
\text { Efficiency }\end{array}$} & \multicolumn{2}{|c|}{ Performance } \\
\hline & & & & & & Initial & Final \\
\hline $\begin{array}{l}\text { Mezzaroba } \\
\text { and Machado } \\
\text { (2014) }\end{array}$ & $\begin{array}{l}\text { To determine the influence of age, anthropometry, } \\
\text { and distance on stroke parameters and } \\
\text { performance }\end{array}$ & Cross-sectional & $\begin{array}{l}\text { BM, BF, H, TUEL, } \\
\text { TLEL }\end{array}$ & V, SF, SL & $\mathrm{SI}$ & \multicolumn{2}{|c|}{$\begin{array}{l}10-11 \text { years } 100 \mathrm{~m} \text { Free: } 1.10 \pm 0.17 \mathrm{~m} \cdot \mathrm{s}^{-1} \\
10-11 \text { years } 200 \mathrm{~m} \text { Free: } 1.02 \pm 0.15 \mathrm{~m} \cdot \mathrm{s}^{-1} \\
10-11 \text { years } 400 \mathrm{~m} \text { Free: } 0.95 \pm 0.14 \mathrm{~m} \cdot \mathrm{s}^{-1} \\
12-13 \text { years } 100 \mathrm{~m} \text { Free: } 1.28 \pm 0.12 \mathrm{~m} \cdot \mathrm{s}^{-1} \\
12-13 \text { years } 200 \mathrm{~m} \text { Free: } 1.14 \pm 0.12 \mathrm{~m} \cdot \mathrm{s}^{-1} \\
12-13 \text { years } 400 \mathrm{~m} \text { Free: } 1.07 \pm 0.14 \mathrm{~m} \cdot \mathrm{s}^{-1}\end{array}$} \\
\hline $\begin{array}{l}\text { Morais et al. } \\
(2012)\end{array}$ & $\begin{array}{l}\text { To develop a structural equation model for } \\
\text { performance in young swimmers based on } \\
\text { selected kinematic, anthropometric and } \\
\text { hydrodynamic variables }\end{array}$ & Cross-sectional & $\mathrm{BM}, \mathrm{H}, \mathrm{AS}, \mathrm{HSA}$ & $S L, d v, D_{a}$ & $\mathrm{SI}$ & \multicolumn{2}{|c|}{$\begin{array}{l}\text { Boys } 100 \text { Free: } 78.33 \pm 12.07 \mathrm{~s} \\
\text { Girls } 100 \text { Free: } 85.25 \pm 13.89 \mathrm{~s} \\
\text { Together } 100 \text { Free: } 82.07 \pm 12.96 \mathrm{~s}\end{array}$} \\
\hline $\begin{array}{l}\text { Morais et al. } \\
\text { (2013a) }\end{array}$ & $\begin{array}{l}\text { To analyze a gender and sports level effect, and } \\
\text { sports level-gender interactions on } \\
\text { anthropometrics, kinematics and energetics }\end{array}$ & Cross-sectional & $\begin{array}{l}\text { BM, H, AS, TTSA, } \\
\text { HSA, FSA }\end{array}$ & $v, S L, S F, d v$ & $\mathrm{SI}, \mathrm{CV}, \eta_{\mathrm{F}}$ & \multicolumn{2}{|c|}{$\begin{array}{l}\text { Swimmers were faster in Tier and performance decreased until Tier } 4 \\
\text { (for boys only and girls only) }\end{array}$} \\
\hline $\begin{array}{l}\text { Morais et al. } \\
(2013 b)\end{array}$ & $\begin{array}{l}\text { To follow-up the stability of performance and its } \\
\text { determinant factors (i.e., } \\
\text { anthropometrics, kinematics, hydrodynamics } \\
\text { and efficiency) }\end{array}$ & $\begin{array}{l}\text { Longitudinal (one } \\
\text { competitive } \\
\text { season) }\end{array}$ & $\begin{array}{l}\text { BM, H, AS, TTSA, } \\
\text { HSA, FSA, CP }\end{array}$ & $\begin{array}{l}D_{a}, C_{D a}, v, S L, S F \\
d v\end{array}$ & $\mathrm{SI}, \eta_{\mathrm{F}}$ & \multicolumn{2}{|c|}{$\begin{array}{l}\text { Performance improved significantly between the three evaluation moments } \\
\text { (for boys and girls pooled together and individually) }\end{array}$} \\
\hline $\begin{array}{l}\text { Morais et al. } \\
\text { (2014a) }\end{array}$ & $\begin{array}{l}\text { To model a latent growth curve of } \\
\text { the performance and biomechanics }\end{array}$ & $\begin{array}{l}\text { Longitudinal (one } \\
\text { competitive } \\
\text { season) }\end{array}$ & n.a. & $D_{a}, C_{D a}, P_{d}, S F, d v$ & $\eta_{\mathrm{F}}$ & 100 Free: $72.05 \pm 5.33 \mathrm{~s}$ & 100 Free: $66.13 \pm 5.16 \mathrm{~s}$ \\
\hline $\begin{array}{l}\text { Morais et al. } \\
\text { (2014b) }\end{array}$ & $\begin{array}{l}\text { To assess the intra- and inter-individual variability } \\
\text { of the performance and its determinant factors } \\
\text { within and between seasons according to gender } \\
\text { and skill level }\end{array}$ & $\begin{array}{l}\text { Longitudinal (two } \\
\text { competitive } \\
\text { seasons) }\end{array}$ & $\begin{array}{l}\text { BM, H, AS, TTSA, } \\
\text { HSA, FSA, CP }\end{array}$ & $\begin{array}{l}\mathrm{D}_{\mathrm{a}}, \mathrm{C}_{\mathrm{Da}}, \mathrm{v}, \mathrm{SL}, \mathrm{SF} \\
\mathrm{dv}\end{array}$ & $\mathrm{SI}, \eta_{\mathrm{F}}$ & \multicolumn{2}{|c|}{$\begin{array}{c}\text { Boys (high skill) } 100 \text { Free: } \Delta=13.39 \% \\
\text { Boys (average skill) } 100 \text { Free: } \Delta=27.80 \% \\
\text { Girls (high skill) } 100 \text { Free: } \Delta=7.77 \% \\
\text { Girls (average skill) } 100 \text { Free: } \Delta=17.85 \%\end{array}$} \\
\hline $\begin{array}{l}\text { Morais et al. } \\
(2015)\end{array}$ & $\begin{array}{l}\text { To apply a new method to identify, classify, and } \\
\text { follow up swimmers, based on their performance } \\
\text { and its determinant factors, and to analyze the } \\
\text { swimmers'stability over a competitive season with } \\
\text { that method }\end{array}$ & $\begin{array}{l}\text { Longitudinal (one } \\
\text { competitive } \\
\text { season) }\end{array}$ & $\mathrm{AS}, \mathrm{CP}$ & $C_{D a}, v, d v, S L$ & $\mathrm{SI}, \eta_{\mathrm{F}}$ & $\begin{array}{l}\text { High skill } 100 \text { Free: } 71.17 \pm 5.91 \mathrm{~s} \\
\text { Average skill } 100 \text { Free: } 77.57 \pm 4.44 \mathrm{~s} \\
\text { Low skill } 100 \text { Free: } 83.67 \pm 5.11 \mathrm{~s}\end{array}$ & $\begin{array}{l}\text { High skill } 100 \text { Free: } 61.63 \pm 2.90 \mathrm{~s} \\
\text { Average skill } 100 \text { Free: } \\
68.64 \pm 3.36 \mathrm{~s} \text { Low skill } 100 \text { Free: } \\
73.43 \pm 3.92 \mathrm{~s}\end{array}$ \\
\hline $\begin{array}{l}\text { Morais et al. } \\
\text { (2016) }\end{array}$ & $\begin{array}{l}\text { To compute a confirmatory model for } \\
\text { swimming performance based on } \\
\text { anthropometrics, strength, power output, } \\
\text { kinematics, and efficiency. }\end{array}$ & Cross-sectional & $\mathrm{BM}, \mathrm{H}, \mathrm{AS}$ & $\mathrm{BT}, \mathrm{v}, \mathrm{P}_{\mathrm{d}}$, & $\eta_{\mathrm{F}}$ & \multicolumn{2}{|c|}{100 Free: $74.25 \pm 8.80 \mathrm{~s}$} \\
\hline $\begin{array}{l}\text { Morais et al. } \\
(2017)\end{array}$ & $\begin{array}{l}\text { To test a performance-predictor model based on } \\
\text { swimmers' biomechanical profile, relate the partial } \\
\text { contribution of the main predictors with the } \\
\text { training program over time, and analyze the } \\
\text { time effect, sex effect, and time } \times \text { sex interaction }\end{array}$ & $\begin{array}{l}\text { Longitudinal } \\
\text { (three competitive } \\
\text { seasons) }\end{array}$ & $\mathrm{BM}, \mathrm{H}, \mathrm{AS}$ & $S F, S L, v, d v$ & $\mathrm{SI}, \eta_{\mathrm{F}}$ & $\begin{array}{l}\text { Boys } 100 \text { Free: } 76.26 \pm 7.00 \mathrm{~s} \\
\text { Girls } 100 \text { Free: } 79.06 \pm 6.77 \mathrm{~s}\end{array}$ & $\begin{array}{l}\text { Boys } 100 \text { Free: } 60.08 \pm 3.22 \mathrm{~s} \text { Girls } \\
100 \text { Free: } 68.06 \pm 4.40 \mathrm{~s}\end{array}$ \\
\hline $\begin{array}{l}\text { Morais et al. } \\
\text { (2020a) }\end{array}$ & $\begin{array}{l}\text { To analyze the variations in } \\
\text { performance, anthropometrics, and biomechanics } \\
\text { break to gather insights on the detraining process }\end{array}$ & $\begin{array}{l}\text { Longitudinal } \\
\text { (11 weeks) }\end{array}$ & $\begin{array}{l}\text { BM, H, AS, TTSA, } \\
\text { HSA, FSA }\end{array}$ & $\begin{array}{l}D_{a}, C_{D a}, v, S L, S F \\
d v, P_{d}, P_{k}, P_{\text {ext }}, \\
E_{\text {tot }}, F_{r}, v_{h}, R_{e}\end{array}$ & $\mathrm{SI}, \eta_{\mathrm{F}}$ & $\begin{array}{l}\text { Boys } 100 \text { Free: } 68.53 \pm 6.81 \mathrm{~s} \\
\text { Girls } 100 \text { Free: } 75.07 \pm 7.84 \mathrm{~s}\end{array}$ & $\begin{array}{c}\text { Boys } 100 \text { Free: } 70.05 \pm 5.84 \mathrm{~s} \text { Girls } \\
100 \text { Free: } 76.53 \pm 6.44 \mathrm{~s}\end{array}$ \\
\hline $\begin{array}{l}\text { Morais et al. } \\
\text { (2020b) }\end{array}$ & $\begin{array}{l}\text { To classify, identify and follow-up swimmers into } \\
\text { sub-groups (clusters), according to the } \\
\text { performance and its biomechanical determinants, } \\
\text { and analyze the individualvariations of each } \\
\text { swimmer }\end{array}$ & $\begin{array}{l}\text { Longitudinal (two } \\
\text { competitive } \\
\text { seasons) }\end{array}$ & $\begin{array}{l}\text { BM, H, AS, TTSA, } \\
\text { HSA, FSA, CP }\end{array}$ & $\begin{array}{l}D_{a}, C_{D a}, v, S L, S F \\
d v, P_{d}, P_{k}, P_{\text {ext }}\end{array}$ & $\mathrm{SI}, \eta_{\mathrm{F}}$ & $\begin{array}{l}\text { High skill } 100 \text { Free: } 68.07 \pm 6.62 \mathrm{~s} \\
\text { Average skill } 10 \text { Free: } 73.14 \pm 4.87 \mathrm{~s} \\
\text { Low skill } 100 \text { Free: } 82.60 \pm 4.18 \mathrm{~s}\end{array}$ & $\begin{array}{c}\text { High skill } 100 \text { Free: } 61.46 \pm 3.43 \mathrm{~s} \\
\text { Average skill } 100 \text { Free: } \\
65.33 \pm 2.97 \mathrm{~s} \text { Low skill } 100 \text { Free: } \\
70.09 \pm 3.48 \mathrm{~s}\end{array}$ \\
\hline $\begin{array}{l}\text { Moreira et al. } \\
\text { (2014) }\end{array}$ & $\begin{array}{l}\text { To analyze the effects of growth on swimmers' } \\
\text { biomechanical profile }\end{array}$ & $\begin{array}{l}\text { Longitudinal } \\
\text { (10 weeks) }\end{array}$ & $\begin{array}{l}\text { BM, H, AS, HSA, } \\
\text { FSA }\end{array}$ & $\mathrm{D}_{\mathrm{a}}, \mathrm{C}_{\mathrm{Da}}, \mathrm{v}, \mathrm{SL}, \mathrm{SF}$ & $\mathrm{SI}, \eta_{\mathrm{F}}$ & \multicolumn{2}{|c|}{ Performance (swim speed) significantly increased } \\
\hline
\end{tabular}


Performance

\begin{tabular}{|c|c|c|c|c|c|c|c|}
\hline Source & Purpose & Design & Anthropometrics & Biomechanics & $\begin{array}{l}\text { Energetics/ } \\
\text { Efficiency }\end{array}$ & Initial & Final \\
\hline $\begin{array}{l}\text { Nevill et al. } \\
\text { (2020) }\end{array}$ & $\begin{array}{l}\text { To explore which key somatic and demographic } \\
\text { characteristics are common to all swimmers and } \\
\text { identify further characteristics that benefit only } \\
\text { specific strokes }\end{array}$ & Cross-sectional & $\begin{array}{l}\text { BM, H, AS, BF, SH, } \\
\text { ULL, UAL, LAL, HL, } \\
\text { LLL, TL, LL, FL, } \\
\text { ARG, FG, WG, TG, } \\
\text { Calf G, AG, Biacr B, } \\
\text { Bililiac B }\end{array}$ & v & n.a. & \multicolumn{2}{|c|}{$\begin{array}{l}\text { Boys } 100 \text { Breast: } 97.70 \pm 13.50 \mathrm{~s} \\
\text { Girls } 100 \text { Breast: } 95.40 \pm 9.50 \mathrm{~s} \\
\text { Girls } 100 \text { Back: } 79.50 \pm 5.00 \mathrm{~s}\end{array}$} \\
\hline $\begin{array}{l}\text { Ozeker et al. } \\
\text { (2020) }\end{array}$ & $\begin{array}{l}\text { To examine the effect of dry-land training in } \\
\text { addition to swimming training on girl's strength } \\
\text { and swimming performance }\end{array}$ & $\begin{array}{l}\text { Longitudinal } \\
\text { (8 weeks) }\end{array}$ & n.a. & $\begin{array}{l}\text { v, SFlexion, SAbd, } \\
\text { EExt, EFlex, HExt, } \\
\text { HAbd, KFE, SAdd }\end{array}$ & CV & $\begin{array}{l}50 \text { Free CG: } 45.71 \pm 7.44 \mathrm{~s} \\
50 \text { Free EG: } 35.24 \pm 2.57 \mathrm{~s} \\
\text { 400 Free CG: } 514.07 \pm 92.58 \mathrm{~s} \\
\text { 400 Free EG: } 352.57 \pm 23.79 \mathrm{~s}\end{array}$ & $\begin{array}{c}50 \text { Free CG: } 45.65 \pm 7.42 \text { s } 50 \text { Free } \\
\text { EG: } 34.25 \pm 2.39 \text { s } 400 \text { Free CG: } \\
513.04 \pm 92.98 \text { s } 400 \text { Free EG: } \\
343.98 \pm 22.10 \mathrm{~s}\end{array}$ \\
\hline $\begin{array}{l}\text { Poujade et al. } \\
\text { (2003) }\end{array}$ & $\begin{array}{l}\text { To define the determining factors } 400 \mathrm{~m} \\
\text { performance }\end{array}$ & Cross-sectional & $\mathrm{BM}, \mathrm{BF}, \mathrm{H}, \mathrm{AS}, \mathrm{BSA}$ & v & $\mathrm{C}_{\mathrm{s}}, \mathrm{VO}_{2}$ & \multicolumn{2}{|c|}{$400 \mathrm{~m}$ Free: $335.00 \pm 10.00 \mathrm{~s}$} \\
\hline $\begin{array}{l}\text { Poujade et al. } \\
\text { (2002) }\end{array}$ & $\begin{array}{l}\text { To measure the Cs and to examine the } \\
\text { relationship between Cs and velocity, morphology } \\
\text { and stroking parameters }\end{array}$ & Cross-sectional & $\begin{array}{l}\text { BM, BF, H, BSA, } \\
\text { HLift }\end{array}$ & SF, SL, v & $\begin{array}{l}\mathrm{C}_{\mathrm{s}}, \mathrm{C}_{\mathrm{S}} / \mathrm{SA} \\
\mathrm{C}_{\mathrm{S}} / \mathrm{SA}, \mathrm{HL} \\
\mathrm{VO}_{2}\end{array}$ & \multicolumn{2}{|c|}{$400 \mathrm{~m}$ Free: $335.77 \pm 9.77 \mathrm{~s}$} \\
\hline $\begin{array}{l}\text { Saavedra } \\
\text { et al. (2013) }\end{array}$ & $\begin{array}{l}\text { To determine the volume of training, how it } \\
\text { evolves and its relationship with performance }\end{array}$ & Cross-sectional & $\mathrm{BM}, \mathrm{H}, \mathrm{SH}, \mathrm{AS}$ & n.a. & n.a. & \multicolumn{2}{|c|}{ n.a. } \\
\hline $\begin{array}{l}\text { Saavedra } \\
\text { et al. (2010) }\end{array}$ & $\begin{array}{l}\text { To analyze swimming performance by developing } \\
\text { multivariate predictive modelsbased on a wide } \\
\text { variety of assessments from a } \\
\text { multidimensional perspective }\end{array}$ & Cross-sectional & $\begin{array}{l}\text { BM, BF, BMI, H, } \\
\text { SH, AS, HL, HW, } \\
\text { FL, FW, Biacr } \\
\text { B,Biiliac B, Bitroch } \\
\text { B, KB, EB, WB, } \\
\text { CG, AFG, GG, TG, } \\
\text { LG, AS/H,Biacr } \\
\text { B/H, CG/H, } \\
\text { GG/H, } \\
\text { SSS }\end{array}$ & $\begin{array}{l}\text { HJ, HG, AFlex, } \\
\text { SFlex, Glide, SF, } \\
\text { SL, v }\end{array}$ & $\begin{array}{l}\text { SRE, FB, PT, } \\
\text { SandR, SR, } \\
\text { Abd, FAH, SI }\end{array}$ & \multicolumn{2}{|c|}{ n.a. } \\
\hline $\begin{array}{l}\text { Sammoud } \\
\text { et al. (2018) }\end{array}$ & $\begin{array}{l}\text { To use allometric models to estimate the optimal } \\
\text { body size, limb segment length, and girth and } \\
\text { breadth ratios associated with } 100-\mathrm{m} \\
\text { breaststroke speed performance }\end{array}$ & Cross-sectional & $\begin{array}{l}\text { APHV, BM, H, AS, } \\
\text { SH, BF, FM, FFM, } \\
\text { BMI, ULL, UAL, } \\
\text { LAL, HL, LLL, TL, } \\
\text { LL, FL, ARG, FG, } \\
\text { WG, TG, Calf G, } \\
\text { AG, Biacr B, Bililiac } \\
\text { B }\end{array}$ & $v$ & n.a. & \multicolumn{2}{|c|}{$\begin{array}{l}\text { Boys } 100 \text { Breast: } 97.70 \pm 13.40 \mathrm{~s} \\
\text { Girls } 100 \text { Breast: } 95.40 \pm 9.50 \mathrm{~s}\end{array}$} \\
\hline $\begin{array}{l}\text { Sammoud } \\
\text { et al. (2019) }\end{array}$ & $\begin{array}{l}\text { To examine the effects of plyometric jump } \\
\text { program in combination with swimming compared } \\
\text { with swimming only on proxies of muscle power }\end{array}$ & $\begin{array}{l}\text { Longitudinal } \\
\text { (8 weeks) }\end{array}$ & $\mathrm{APHV}, \mathrm{BM}, \mathrm{H}$ & $\begin{array}{l}\text { CMJ, SLJ, } 25 \mathrm{~m} \\
\text { KWP, } 25 \mathrm{~m} \text { Free } \\
\text { WP, } v\end{array}$ & n.a. & $\begin{array}{l}\text { CG } 15 \text { Free: } 9.53 \pm 0.80 \mathrm{~s} \\
\text { CG } 25 \text { Free: } 17.17 \pm 1.20 \mathrm{~s} \\
\text { CG } 50 \text { Free: } 37.50 \pm 2.80 \mathrm{~s} \\
\text { EG } 15 \text { Free: } 10.10 \pm 0.50 \mathrm{~s} \\
\text { EG } 25 \text { Free: } 18.20 \pm 0.90 \mathrm{~s} \\
\text { EG } 50 \text { Free: } 40.00 \pm 1.70 \mathrm{~s}\end{array}$ & $\begin{array}{c}\text { CG } 15 \text { Free: } 9.30 \pm 0.80 \text { s CG } 25 \\
\text { Free: } 16.90 \pm 1.40 \text { s CG } 50 \text { Free: } \\
37.60 \pm 4.00 \text { s EG } 15 \text { Free: } \\
9.60 \pm 0.40 \text { s EG } 25 \text { Free: } \\
17.52 \pm 0.70 \text { s EG } 50 \text { Free: } \\
39.10 \pm 1.50 \mathrm{~s}\end{array}$ \\
\hline $\begin{array}{l}\text { Sammoud } \\
\text { et al. (2021) }\end{array}$ & $\begin{array}{l}\text { To examine the effects of an 8-week plyometric } \\
\text { jump training program on jump and sport-specific } \\
\text { performances inprepubertal femaleswimmers }\end{array}$ & $\begin{array}{l}\text { Longitudinal } \\
\text { (8 weeks) }\end{array}$ & APHV, BM, H, BMI & CMJ, SLJ & n.a. & $\begin{array}{l}\text { CG } 25 \text { Free: } 18.35 \pm 1.19 \mathrm{~s} \\
\text { CG } 50 \text { Free: } 40.51 \pm 3.10 \mathrm{~s} \\
\text { EG } 25 \text { Free: } 19.27 \pm 1.13 \mathrm{~s} \\
\text { EG } 50 \text { Free: } 42.79 \pm 2.65 \mathrm{~s}\end{array}$ & $\begin{array}{c}\text { CG } 25 \text { Free: } 18.50 \pm 0.17 \text { s CG } 50 \\
\text { Free: } 40.94 \pm 0.59 \text { s EG } 25 \text { Free: } \\
18.05 \pm 0.15 \mathrm{~s} \text { EG } 50 \text { Free: } \\
41.08 \pm 0.52 \mathrm{~s}\end{array}$ \\
\hline
\end{tabular}


Performance

\begin{tabular}{|c|c|c|c|c|c|c|c|}
\hline Source & Purpose & Design & Anthropometrics & Biomechanics & $\begin{array}{l}\text { Energetics/ } \\
\text { Efficiency }\end{array}$ & Initial & Final \\
\hline $\begin{array}{l}\text { Seffrin et al. } \\
\text { (2021) }\end{array}$ & $\begin{array}{l}\text { To evaluate the characteristics of body, } \\
\text { anthropometry, and neuromuscular fitness in } \\
\text { young swimmers from } 11 \text { to } 23 \text { years old, and fit } \\
\text { multiple regression models to verify which } \\
\text { evaluated factors better explain performance in } \\
100 \text { and } 400 \mathrm{~m} \text { Freestyle }\end{array}$ & Cross-sectional & $\begin{array}{l}\text { BM, LBM, H, AS, } \\
\text { SH, ULL, LLL, FL, } \\
\text { HL, TTSA, TW }\end{array}$ & $\begin{array}{l}\text { CMJ, SJ, HG, } \\
\text { AvgPext, AvgPflex, } \\
\text { PText, PTflex, } \\
\text { AvgPer, AvgPir, } \\
\text { PTer, PTir }\end{array}$ & n.a. & $\begin{array}{l}\text { Boys } 100 \mathrm{~m} \text { Free: } 84.73 \pm 11.15 \mathrm{~s} \\
\text { Boys } 400 \mathrm{~m} \text { Free: } 393.35 \pm 62.93 \mathrm{~s} \\
\text { Girls } 100 \mathrm{~m} \text { Free: } 81.11 \pm 8.45 \mathrm{~s} \\
\text { Girls } 400 \mathrm{~m} \text { Free: } 376.65 \pm 32.52 \mathrm{~s}\end{array}$ & \\
\hline $\begin{array}{l}\text { Silva et al. } \\
\text { (2012) }\end{array}$ & $\begin{array}{l}\text { To characterize the backstroke swimming } \\
\text { technique through the stroke parameters and the } \\
\text { inter-arm coordination }\end{array}$ & Cross-sectional & $\mathrm{BM}, \mathrm{H}, \mathrm{AS}$ & $\begin{array}{l}\text { v, SF, SL, SL/AS, } \\
\text { IdC }\end{array}$ & SI & $\begin{array}{l}\text { Boys } 25 \mathrm{~m} \text { Back: } 1.18 \pm 0.14 \mathrm{~m} \cdot \mathrm{s}^{-1} \\
\text { Girls } 25 \mathrm{~m} \text { Back: } 1.06 \pm 0.14 \mathrm{~m} \cdot \mathrm{s}^{-1}\end{array}$ & \\
\hline $\begin{array}{l}\text { Silva et al. } \\
\text { (2013) }\end{array}$ & $\begin{array}{l}\text { To characterize the front crawl technique by } \\
\text { assessing the general biomechanical parameters } \\
\text { and the inter-arm coordination }\end{array}$ & Cross-sectional & $\mathrm{BM}, \mathrm{H}, \mathrm{AS}$ & $\begin{array}{l}\text { v, SF, SL, SL/AS, } \\
\text { IdC }\end{array}$ & SI & $\begin{array}{l}\text { Boys } 25 \mathrm{~m} \text { Free: } 1.46 \pm 0.12 \mathrm{~m} \cdot \mathrm{s}^{-1} \\
\text { Girls } 25 \mathrm{~m} \text { Free: } 1.37 \pm 0.18 \mathrm{~m} \cdot \mathrm{s}^{-1}\end{array}$ & \\
\hline $\begin{array}{l}\text { Staub et al. } \\
\text { (2020b) }\end{array}$ & $\begin{array}{l}\text { To explore how consistent career pathways } \\
\text { develop among age group swimmers }\end{array}$ & $\begin{array}{l}\text { Longitudinal } \\
\text { (8 years) }\end{array}$ & n.a. & n.a. & n.a. & n.a. & \\
\hline $\begin{array}{l}\text { Staub et al. } \\
\text { (2020a) }\end{array}$ & $\begin{array}{l}\text { To investigate within-sport specialization and entry } \\
\text { age in the careers of German age-group } \\
\text { swimmers }\end{array}$ & $\begin{array}{l}\text { Longitudinal } \\
\text { (8 years) }\end{array}$ & n.a. & n.a. & n.a. & n.a. & \\
\hline $\begin{array}{l}\text { Tijani et al. } \\
\text { (2019) }\end{array}$ & $\begin{array}{l}\text { To investigate the relationship between } \\
\text { anthropometrical and stroking parameters and } \\
\text { their contribution to sprint swimming performance }\end{array}$ & Cross-sectional & $\begin{array}{l}\mathrm{BM}, \mathrm{H}, \mathrm{AS}, \mathrm{AS} / \mathrm{H}, \\
\mathrm{BMI}, \mathrm{BF}\end{array}$ & $v, S F, S L$ & SI & $50 \mathrm{~m}$ Free: $31.27 \pm 1.10 \mathrm{~s}$ & \\
\hline $\begin{array}{l}\text { Tsalis et al. } \\
\text { (2012) }\end{array}$ & $\begin{array}{l}\text { To examine the physiological responses, the } \\
\text { strokeparameter changes and the ability to } \\
\text { sustain a velocity corresponding to critical velocity } \\
\text { during interval swimming }\end{array}$ & Cross-sectional & BF, FM, LBM, S9 & $v, S F, S L$ & $\begin{array}{l}\mathrm{HR}, \mathrm{CV}, \mathrm{CSR} \\
\mathrm{Bl}\end{array}$ & $\begin{array}{l}\text { Children } 50 \mathrm{~m}: 37.70 \pm 1.50 \mathrm{~s} \\
\text { Young } 50 \mathrm{~m}: 32.40 \pm 1.30 \mathrm{~s} \\
\text { Adult } 50 \mathrm{~m}: 31.10 \pm 2.20 \mathrm{~s} \\
\text { Children } 100 \mathrm{~m}: 85.70 \pm 4.80 \mathrm{~s} \\
\text { Young } 100 \mathrm{~m}: 71.50 \pm 2.90 \mathrm{~s} \\
\text { Adult } 100 \mathrm{~m}: 68.20 \pm 3.60 \mathrm{~s} \\
\text { Children } 200 \mathrm{~m}: 191.80 \pm 10.40 \mathrm{~s} \\
\text { Young } 200 \mathrm{~m}: 157.90 \pm 9.20 \mathrm{~s} \\
\text { Adult } 200 \mathrm{~m}: 151.30 \pm 5.60 \mathrm{~s} \\
\text { Children } 400 \mathrm{~m}: 400.40 \pm 18.9 \mathrm{~s} \\
\text { Young } 400 \mathrm{~m}: 332.30 \pm 23.00 \mathrm{~s} \\
\text { Adult } 400 \mathrm{~m}: 315.20 \pm 14.60 \mathrm{~s}\end{array}$ & \\
\hline $\begin{array}{l}\text { Zarzeczny } \\
\text { et al. (2013) }\end{array}$ & $\begin{array}{l}\text { To find out if critical swim speed estimated on the } \\
\text { basis of two distances ( } 50 \text { and } 400 \mathrm{~m} \text { ) } \\
\text { corresponds to the results obtained during a } \\
\text { standard 12-minute swim test }\end{array}$ & Cross-sectional & $\mathrm{BM}, \mathrm{H}$ & $v$ & $\begin{array}{l}\text { CV, HR rest, } \\
\text { RR sys, RR } \\
\text { diast }\end{array}$ & $\begin{array}{l}12 \mathrm{~min} \text { test Free: } 0.85 \pm 0.03 \mathrm{~m} \cdot \mathrm{s}^{-1} \\
12 \mathrm{~min} \text { test Breast: } 0.73 \pm 0.02 \mathrm{~m} \cdot \mathrm{s}^{-1}\end{array}$ & \\
\hline
\end{tabular}

n.a. - not applicable (i.e., not reported).

Free, freestyle; back, backstroke; breast, breaststroke; fly, butterfly; Acad_level, the academic level of coaches (1, bachelor; 2, master; 3, philosophy doctor); coach level, the training level of coaches (1, level 1; 2, level 2; 3, level 3); Exp, training experience of coaches ( $\leq$, equal or less than 5 years; > 5, more than 5 years); CG, control group; EG, experimental group; U13, under the 13-year level; U12, under the 12-year level. 
of average age), including the freestyle and butterfly strokes (Nevill et al., 2020).

As young swimmers grow until reaching full maturity, the best way to gather deeper insights into the influence of anthropometrics on swimming performance is to design longitudinal studies (Lätt et al., 2009a,b; Abbott et al., 2021). When following up over a competitive season, swimmers who achieved better performances (in the $100-\mathrm{m}$ freestyle) also had larger body sizes (Morais et al., 2020b). A similar trend was verified in the 400-m freestyle (Lätt et al., 2009a,b). Moreover, a 3-year study that recruited 91 swimmers from a TID program showed that the AS was a major cause of performance improvement (Morais et al., 2017). Nonetheless, it was argued that swimmers must "relearn" the stroke mechanics to better use the propelling limbs, whenever meaningful body changes happen, such as during growth spurts (Morais et al., 2017). This happens because, as mentioned earlier, anthropometry not only has a direct effect on the performance of swimmers but also holds a concurrent effect on other scientific domains related to swimming techniques (Tijani et al., 2019; Morais et al., 2020b). That is, longer lengths like $\mathrm{H}$ and AS are strongly related to longer stroke length (SL) (kinematics) (Silva et al., 2012; Morais et al., 2017); whereas, larger TTSA or BSA is strongly related to more drag (hydrodynamics) (Barbosa et al., 2014).

Young swimmers are prone to have several growth spurts within a competitive season (Abbott et al., 2021). Such spurts contribute to the improvement in several variables related to swimming technique (Morais et al., 2013b, 2015). It was shown that, even during detraining periods (i.e., training breaks) the performance impaired, but anthropometry was responsible for slowing down such impairment (Moreira et al., 2014; Morais et al., 2020a). That is, during an 11-week detraining period, the swimmers continued to grow up. Because they were taller at the end of the break, it allowed them to minimize the performance impairment (Morais et al., 2020a). This highlights the importance of a systematic and frequent assessment of the anthropometrics.

\section{Biomechanics}

Biomechanics is related to swimming techniques, such as SL, stroke frequency (SF), stroke index (SI), and intra-cyclic variation of the swim speed $(\mathrm{dv})$, which are part of the "nurture" process and the ones that better explain performance (Lätt et al., 2009a; Barbosa et al., 2010; Morais et al., 2012). Top-tier swimmers are faster, because of better SL, SF, Reynolds number $\left(\mathrm{R}_{\mathrm{e}}\right)$, Froude number $\left(\mathrm{F}_{\mathrm{r}}\right)$, and hull speed $\left(\mathrm{V}_{\mathrm{h}}\right)$ scores (Barbosa et al., 2019). Faster swimmers were also prone to have less dv (Barbosa et al., 2014; Figueiredo et al., 2016) and deliver more in-water mechanical power (Barbosa et al., 2015, 2019; Morais et al., 2020b). Thus, it seems that the fastest swimmers can promote smaller speed fluctuations (Barbosa et al., 2014) and produce more power concurrently (Barbosa et al., 2019; Morais et al., 2020b). It can be argued that in-water power is related to more dry-land strength. It has been shown that variables related to dryland strength were correlated with sprint swimming (Garrido et al., 2010a; Seffrin et al., 2021) and middle-distance events (400-m freestyle-Seffrin et al., 2021). Moreover, the power to overcome drag can be explained by $94 \%$ of the dry-land strength (Morais et al., 2016). However, faster swimmers are also under more active drag $\left(D_{a}\right)$ and coefficient of active drag $\left(C_{D a}\right)$ (Barbosa et al., 2019). It should be noted that drag variables, such as $D_{a}$, passive drag $\left(D_{p}\right), C_{D a}$, and coefficient of passive drag $\left(\mathrm{C}_{\mathrm{Dp}}\right)$, are highly dependent on velocity, TTSA, and BSA (Kjendlie and Stallman, 2008). Thus, bigger and faster swimmers are prone to be under more drag (Barbosa et al., 2014, 2019). Indeed, "matured" age-group swimmers performing freestyle (Silva et al., 2012) and backstroke (Silva et al., 2013) had higher stroke kinematics scores [namely swim speed (v) and SL]. Conversely, non-significant differences were found in the index of coordination (IdC) (i.e., motor control) between pre and postpubertal swimmers (Silva et al., 2012, 2013).

Longitudinal studies showed that variables related to biomechanics change significantly over time (Lätt et al., 2009a; Morais et al., 2015, 2020b). As aforementioned, young swimmers undergo growth and maturation processes that lead to changes in the swimming technique (Lätt et al., 2009a; Morais et al., 2017). They are prone to improve the kinematics and kinetics over longterm periods of time (Morais et al., 2017, 2020b). Nonetheless, in specific moments of a season, young swimmers may impair the stroke biomechanics (Morais et al., 2013b, 2014b). Despite the variations within the season, swimmers improved the stroke biomechanics when comparing the beginning and the end of the season. Longitudinal research also reported that swimmers cluster in groups with similar traits related to stroke biomechanics (Morais et al., 2015, 2020b). As far as the long term is concerned, i.e., during one or several competitive seasons, the variables that better characterize each group may change over time. Swimmers improve and impair the stroke biomechanics several times over one or more competitive seasons (Morais et al., 2015, 2020b). Notwithstanding, variations may not occur at the same time across all clusters (Morais et al., 2015, 2020b). Moreover, it has been shown that swimmers are also likely to change groups; that is, switching to another subgroup or performance level. A swimmer who is assigned to the top-tier subgroup may not remain in that subgroup. It is possible that, over the season, the swimmer may drop to a lower tier, and lower-tier swimmers can climb up to top-tier groups (Morais et al., 2020b). Performance levels are very dynamic over time, and swimmers can move to different tiers quite often. The shift is due to a concurrent change in the determinant factors underlying the performance, which, in turn, depend on the developmental training program they are under, as well as the rate of growth and maturation.

The relationship between the in-water training programs and swimming biomechanics can be better understood when internal and external training loads are monitored. However, few studies addressed this topic in developing programs for young swimmers (Garrido et al., 2010b; Saavedra et al., 2013; Morais et al., 2014a). High-training volumes during the first part of a season (with low intensity, including warm-up, recovery, and slow-pace drills) led to an improvement in performance (Morais et al., 2014a). The same authors (Morais et al., 2014a) evaluated a group of swimmers during a competitive season in four different moments. They achieved $59 \%$ of the final performance in the second evaluation moment and $99 \%$ in 
the third moment. Between the 3rd and 4th (final) moments, the swimmers improved by only $1 \%$, with the SF as the main determinant (Morais et al., 2014a). Between the 3rd and 4th moments, the periodization included an increase in the aerobic power and aerobic capacity (Morais et al., 2014a). As their older counterparts, young swimmers increase SF whenever they want to reach faster speeds (Mezzaroba and Machado, 2014; Barbosa et al., 2019). The researchers noted that changes in performance are related to the type of training swimmers were undergoing at the time of each evaluation moment. Thus, coaches can use different training strategies for their periodization to reach previously outlined goals and avoid burnout.

Studies also aimed to understand the effect of dry-land strength on the performance of young swimmers (Sammoud et al., 2019, 2021; Ozeker et al., 2020). During an 8-week intervention (aerobic in-water training concurrently with dryland strength), Garrido et al. (2010b) reported a trend in sprint performance improvement (25- and 50-m freestyle) due to strength training. This was confirmed in other sprint events (50- and 100-m freestyle and backstroke) (Alshdokhi et al., 2020). Swimmers assigned to the experimental group presented a larger increase in the selected variables compared with the control group (Alshdokhi et al., 2020). It was suggested that the improvement in dry-land strength resulted in better swimming performance. Others aimed to provide deeper insights into the effect of different types of dry-land strength and conditioning programs on sprint performance (50-m freestyle) (Amaro et al., 2017). It was noted that swimmers under explosiveness training (i.e., performing the repetition quickly) presented larger improvements in swimming speed compared with performing repetition/sets training (Amaro et al., 2017). The phenomenon of post-activation potentiation performance enhancement is defined as a voluntary dynamic force production after a short and acute bout of high-intensity voluntary exercise (Blazevich and Babault, 2019). A study used three 30-s postactivation potentiation protocols ( $10 \mathrm{~min}$ before competition) to understand its effect on the performance and stroke kinematics (Abbes et al., 2018). Authors verified that all protocols presented non-significant effects on the $50-\mathrm{m}$ freestyle performance, SL, and SF. A follow-up study analyzed the effect of tethered swimming as post-activation potentiation in the $50-\mathrm{m}$ freestyle performance and stroke kinematics (SL), and non-significant effects were observed (Abbes et al., 2020). Therefore, both studies suggest an unclear effect of post-activation potentiation performance enhancement on young swimmers.

\section{Energetics and Efficiency}

Energetics and efficiency also play a role in the performance of young swimmers. That said, the energetic spartial contribution to the performance increases with age (Zacca et al., 2020). It has been observed that $\mathrm{VO}_{2}$ during submaximal swimming speeds is significantly lower in children than adults (Kjendlie et al., 2004a). A study that selected anthropometrics, kinematics, energetics, and efficiency as main outcomes demonstrated that the 100-m freestyle performance was predicted by anaerobic power (AnP), critical velocity (CV), and SI (as an efficiency proxy) (de Mello Vitor and Böhme, 2010).
The CV is a variable commonly used to assess the energetics of young swimmers (Denadai et al., 2000; Marinho et al., 2011; Zarzeczny et al., 2013). It is calculated based on the distancetime slope of several events or swimming distances (Dekerle et al., 2002). It is highly correlated with aerobic performance and, hence, used to control training intensities (Zarzeczny et al., 2013; Figueiredo et al., 2016). However, CV may underestimate swimming intensity corresponding to speed at a blood lactate concentration of $4 \mathrm{mmo} \cdot \mathrm{l}^{-1}$ in swimmers aged 10 to 12 years old (Denadai et al., 2000). It was suggested that it relates, instead, to the intensity corresponding to the maximum steady state of lactate concentration (Denadai et al., 2000). The CV has a significantly direct effect on the 200-m freestyle (Barbosa et al., 2010) and can also provide a strong explanation in the shorter events performances, such as the 100-m freestyle (de Mello Vitor and Böhme, 2010). Swimmers with faster CV also delivered better performances in the 100-m freestyle (Morais et al., 2013a) and 25-m freestyle time trials (Figueiredo et al., 2016).

Besides the SI, researchers also selected the Froude efficiency $\left(\eta_{\mathrm{F}}\right)$ as another energetic proxy (e.g., de Mello Vitor and Böhme, 2010; Morais et al., 2014a). The SI measures the ability of the swimmer to complete a given distance with a particular speed in the fewest possible number of strokes (Costill et al., 1985). The $\eta_{\mathrm{F}}$ estimates the amount of work or power used to translate the body in water (Zamparo et al., 2020). Both variables are straightforward and less time-consuming to compute compared with a direct measurement of other energetics variables (Figueiredo et al., 2016; Barbosa et al., 2019; Morais et al., 2020b). Larger SI and $\eta_{F}$ are associated with better performance in short distances, as the 100-m freestyle and 25$\mathrm{m}$ freestyle time trial. Indeed, the fastest swimmers distinguish themselves from others because they have a better $\mathrm{CV}$, SI, and $\eta \mathrm{F}$ (Morais et al., 2013a; Figueiredo et al., 2016; Barbosa et al., 2019). Moreover, it should be highlighted that the increase in SI and $\eta_{\mathrm{F}}$ is related to the technical training that young swimmers undergo (Morais et al., 2017).

For longer events, such as the $400-\mathrm{m}$ freestyle, the $\mathrm{VO}_{2 \max }$ (Duché et al., 1993; Poujade et al., 2003) and the $\mathrm{VO}_{2 \text { peak }}$ (Jürimäe et al., 2007) were the best predictors of swimming performance within a set of energetic variables. Hue et al. (2013) showed that the fastest swimmers in the 400-m freestyle event also had better $\mathrm{VO}_{2 \max }$ than their slower counterparts. When tested by the $5 \times 300-\mathrm{m}$ protocol, young swimmers improved their swimming economy as they got older based on lower heart rate (HR) variability (Tsalis et al., 2012). In mid-distance events, another variable monitored very frequently was the energy cost of swimming $\left(\mathrm{C}_{\mathrm{s}}\right)$, which increases with swimming speed (Poujade et al., 2002; Kjendlie et al., 2004a,b). Nonetheless, one study pointed out that kinematics (SL and SF), anthropometrics (body length-BL, BM, and BSA), and HL did not explain the $\mathrm{C}_{S}$ in young swimmers (Poujade et al., 2002). The authors suggested that underwater torque, technical ability, and maturation could be strong predictors. Another study reported that passive torque presented a significant linear relationship with absolute $C_{s}$ in young swimmers (Kjendlie et al., 2004b). Overall, there is solid evidence that, for similar swimming speeds, young swimmers have more $\mathrm{C}_{\mathrm{s}}$ than their older counterparts (Zamparo et al., 
2000; Kjendlie et al., 2004a). Thus, the differences between young swimmers and their older counterparts in the economy are due to the less-technical ability of the former ones.

Longitudinal studies showed that an improvement in energetics $\left(\mathrm{VO}_{2}\right.$ and $\left.\Delta \mathrm{La}\right)$ allowed an enhancement in performance (Lätt et al., 2009a,b). These studies were mostly focused on the 400-m freestyle (i.e., middle distance) (Lätt et al., 2009a,b; Ferreira et al., 2019). A research group followed boys (Lätt et al., 2009a) and girls (Lätt et al., 2009b) during two competitive seasons. It was observed that the $\mathrm{VO}_{2}$ was among the best predictors of performances of both sexes. Others noted significant correlations between a set of energetic variables (i.e., $\mathrm{Bl}$ and $\mathrm{Bg}$ ) in the 400-m freestyle performance (Ferreira et al., 2019). Nevertheless, SI (efficiency) was the best predictor of all the variables assessed (Lätt et al., 2009a,b), or the one that presented the highest correlation with performance (Ferreira et al., 2019). Additionally, it was suggested that the 400-m freestyle enhancement during a season was highly related to an increase in the SI, suggesting that, when swimmers are in this age group, coaches should prioritize technical development of the swimmers (Ferreira et al., 2021). That said, the authors indicated that, concurrently, with the technical enhancement, physiological variables are as important to optimize swimming performance in such middle-distance events (Ferreira et al., 2021). Thus, at early ages, training should focus on learning the proper swimming techniques (i.e., technical training).

Nonetheless, the same reasoning (i.e., importance of energetics/efficiency) can be claimed in shorter race events, at least based on research carried out in the 100-m freestyle (Morais et al., 2013b, 2014b). The $\eta_{\mathrm{F}}$ increased or at least was maintained over time (Morais et al., 2020b). Additionally, high skillful swimmers yielded larger efficiency over time compared with their slower counterparts (Morais et al., 2014b, 2015). The HR (as an energetic indicator) may also present an association with the energetics of swimmers in the 50-m, 100-m, (Alshdokhi et al., 2020), and 400-m freestyle (Ferreira et al., 2019). Both studies reported that training has a positive effect on HR of young swimmers. That is, swimmers decreased the HR, suggesting that, for the same task (50-m and 100-m-Alshdokhi et al., 2020; or 400-m freestyle-Ferreira et al., 2019), they required less effort, with improved performance. Therefore, it can be implied that, besides the middle-distance events (i.e., 400-m freestyle), energetics/efficiency also presents a strong contribution in shorter events (like the 50 and 100-m freestyle).

\section{Performance in a Long-Term Athlete Development (LTAD) Perspective}

Longitudinal studies can also help to understand the evolution of swimming performance from childhood to adulthood (Costa et al., 2011; Staub et al., 2020a,b). This research is paramount to better explain how the growth pace of each swimmer affects the performance and its determinant factors (Durand-Bush and Salmela, 2002). As previously noted, the performance level is highly dynamic and depends upon growth and maturation spurts, as well as the development program the swimmer is under. Stability assessment allows the prediction of the future success of young swimmers by the estimation of the performance progression. Based on the analysis of 242 young swimmers (from
12 to 18 years old), a study observed that swimmers should display a 14-19\% improvement from childhood to adulthood in all freestyle events to become part of an elite group (Costa et al., 2011). The same authors also pointed out that the age of 16 is when the ability to predict the adult competitive level increases considerably. Thus, one cannot "neglect" a swimmer who, at a given moment, is slower than his/her peers, because, the following year, he/she can become one of the best in his/her age group (Morais et al., 2015, 2020b).

A study explored how consistent career pathways are among age-group swimmers (Staub et al., 2020b). Swimmers with better FINA points at 11 years old (including events, strokes, and distances) were more likely to be ranked during more years over the analyzed time frame ( 8 years), but the correlation showed a weak effect (Staub et al., 2020b). The authors argued that young swimmers should get the chance to yield from LTAD programs and should not be selected only by their age-group performance level (Staub et al., 2020b). It was claimed that LTAD programs should bring awareness about this phenomenon, which requires advanced understanding from coaches and other practitioners (Lang and Light, 2010).

It has been recently reported that both nature (i.e., anthropometrics) and nurture (i.e., training-namely sports technique) are important to excel in youth swimming (Barbosa et al., 2019). The best performers among three subgroups of swimmers (subgroup \#1: age-group national champions, national record holders or enrolled in talent ID programs) scored very well in variables related to both nature and nurture parameters. Conversely, swimmers in the subgroup \#3 (racing at local competitions) were weaker in both dimensions, and swimmers in the subgroup \#2 (racing at national competitions) showed weaknesses in nature-related factors (i.e., anthropometrics) but were reasonably good in nurture factors (i.e., training). The subgroup \#2 profile shows the potential of swimmers who may be seen as less genetically predisposed, as a result of an effective developmental program (Barbosa et al., 2019; Marinho et al., 2020).

As far as LTAD is concerned, there is also an ongoing dialog about the potential negative effects of large volumes of training in young swimmers (Nugent et al., 2017). Many coaches combine assumptions based on their experience with evidencebased practice. Recently, Marinho et al. (2020) have reported that an improvement in academic degree, coaching level, and coaching experience of the coaches presented a positive and significant contribution to swimming efficiency and performance of young athletes. Swimmers under the guidance of a coach with a higher academic degree, coaching level, or more years of coaching experience were more efficient and, concurrently, delivered better performances (Marinho et al., 2020). As youth swimming training should be focused on technical training (Morais et al., 2012), coaches should be able to provide their athletes with training in key skills and abilities based on such technique determinants. Therefore, age-group coaches are advised to design training programs that are underpinned on high-level and cutting-edge evidence.

Another major topic within LTAD is early specialization (Larson et al., 2019; Staub et al., 2020a). Early specialization refers to young athletes who limit their childhood to a single 
sport, deliberating their training and development on a singular sport (Baker, 2003). It was claimed that early specialization might promote far more risks than benefits (Wiersma, 2000). Youth athletes can suffer from social isolation, overdependence, burnout, manipulation, injury, and compromise their growth and maturation (Malina, 2010). Conversely, an athlete who practices a set of skills with increased frequency and duration becomes more proficient in those skills than one who practices them periodically (Wiersma, 2000). In competitive swimming, there are four competitive swim strokes and one event combining all (medley), as well as several race distances. Thus, in swimming, a within specialization may occur whenever a swimmer chooses and develops at an early age a single stroke or distance (or a combination of more than one stroke or distance, or both combined) (Staub et al., 2020a). A study showed that greater diversification within the same sport is positively correlated with success at the age of 18 (Staub et al., 2020a). Thus, the younger a swimmer enters the top 100 , more likely he/she is to reach a top-tier at the age of 18 (Staub et al., 2020a). This suggests that early specialization may not be the best pathway to ensure higher performance in adulthood. Additionally, Larson et al. (2019) showed that a set of markers related to early specialization was related to burnout or a dropout in youth swimming. However, it was suggested that early specialization in one event, stroke or distance could be a way for coaches to accomplish qualification times and promote rapid adolescent success at the expense of long-term elite success as adults (Staub et al., 2020a). As such, developmental programs should expose young swimmers to a broad range of events (distances and swimming strokes) and even, at early stages, to other aquatic and non-aquatic sports.

\section{CONCLUSIONS}

Performance of young swimmers is characterized by a multifactorial, holistic, and dynamic phenomenon relying

\section{REFERENCES}

Abbes, Z., Chamari, K., Mujika, I., Tabben, M., Bibi, K. W., Hussein, A. M., Martin, C., and Haddad, M. (2018). Do thirty-second post-activation potentiation exercises improve the 50-m freestyle sprint performance in adolescent swimmers? Front Physiol. 9:1464. doi: 10.3389/fphys.2018.01464

Abbes, Z., Haddad, M., Bibi, K. W., Mujika, I., Martin, C., and Chamari, K. (2020). Effect of tethered swimming as postactivation potentiation on swimming performance and technical, hemophysiological, and psychophysiological variables in adolescent swimmers. Int. J. Sports Physiol. Perform. 16, 311-315. doi: 10.1123/ijspp.2019-0669

Abbott, S., Yamauchi, G., Halaki, M., Castiglioni, M. T., Salter, J., and Cobley, S. (2021). Longitudinal relationships between maturation, technical efficiency, and performance in age-group swimmers: improving swimmer evaluation. Int. J. Sports Physiol. Perform. 03:77. doi: 10.1123/ijspp.2020-0377

Alshdokhi, K. A., Petersen, C. J., and Clarke, J. C. (2020). Effect of 8 weeks of grip strength training on adolescent sprint swimming: a randomized controlled trial. Exerc. Med. 4:1. doi: 10.26644/em.2020.001

Amaro, N. M., Marinho, D. A., Marques, M. C., Batalha, N. P., and Morouço, P. G. (2017). Effects of dry-land strength and conditioning programs in age group swimmers. J. Strength Cond. Res. 31, 2447-2454. doi: $10.1519 /$ JSC.0000000000001709 on several features from different scientific domains. Better performance has always been related to better swimming techniques. Concurrently, anthropometry (e.g., higher AS, H, and upper limbs) also plays an important role in performance. Swimmers with larger body dimensions are the fastest. This suggests that anthropometry (i.e., nature) and training (i.e., nurture) play key roles. The contribution of energetics and efficiency becomes more important as the swimmer gets older or whenever the swimming event becomes longer. Performance enhancement of young swimmers should rely on LTAD programs, always taking into consideration the growth spurt and the external training load of the swimmer. Coaches are advised to monitor the rate of growth of their athletes, since this can affect their performance. They should put more focus on improving swimming technique and less on the external training load.

\section{DATA AVAILABILITY STATEMENT}

The datasets presented in this article are not readily available because none. Requests to access the datasets should be directed to Jorge E. Morais, morais.jorgestrela@gmail.com.

\section{AUTHOR CONTRIBUTIONS}

JM, TB, PF, AS, and DM conceived and designed the study. JM, TB, and AS performed the search and data analysis. PF and DM performed the quality assessment. JM carried out the drafting of the manuscript. All authors reviewed the manuscript and approved the submitted version.

\section{FUNDING}

This work is supported by national funds (FCT-Portuguese Foundation for Science and Technology) under the project UIBD/DTP/04045/2020.
Baker, J. (2003). Early specialization in youth sport: a requirement for adult expertise? High. Abil. Stud. 14, 85-94. doi: 10.1080/135981303 04091

Barbosa, T. M., Bartolomeu, R., Morais, J. E., and Costa, M. J. (2019). Skillful swimming in age-groups is determined by anthropometrics, biomechanics and energetics. Front. Physiol. 10:73. doi: 10.3389/fphys.2019.00073

Barbosa, T. M., Costa, M., Marinho, D. A., Coelho, J., Moreira, M., and Silva, A. J. (2010). Modeling the links between young swimmers' performance: energetic and biomechanic profiles. Pediatr. Exerc. Sci. 22, 379-391. doi: 10.1123/pes.22.3.379

Barbosa, T. M., Morais, J. E., Costa, M. J., Goncalves, J., Marinho, D. A., and Silva, A. J. (2014). Young swimmers' classification based on kinematics, hydrodynamics, and anthropometrics. J. Appl. Biomech. 30, 310-315. doi: 10.1123/jab.2013-0038

Barbosa, T. M., Morais, J. E., Marques, M. C., Costa, M. J., and Marinho, D. A. (2015). The power output and sprinting performance of young swimmers. J. Strength Cond. Res. 29, 440-450. doi: 10.1519/JSC.0000000000000626

Bielec, G., and Jurak, D. (2019). The relationship between selected anthropometric variables and the sports results of early pubescent swimmers. Balt. J. Health Phys. Act. 11, 124-130. doi: 10.29359/BJHPA.11.1.13

Blazevich, A. J., and Babault, N. (2019). Post-activation potentiation (PAP) vs. post-activation performance enhancement (PAPE) in humans: Historical 
perspective, underlying mechanisms, and current issues. Front. Physiol. 10:1359. doi: 10.3389/fphys.2019.01359

Blume, K., and Wolfarth, B. (2019). Identification of potential performancerelated predictors in young competitive athletes. Front. Physiol. 10:1394. doi: 10.3389/fphys.2019.01394

Costa, M. J., Marinho, D. A., Bragada, J. A., Silva, A. J., Barbosa, T. M. (2011). Stability of elite freestyle performance from childhood to adulthood. J. Sports Sci. 29, 1183-1189. doi: 10.1080/02640414.2011.587196

Costill, D., Kovaleski, J., Porter, D., Kirwan, J., Fielding, R., and King, D. (1985). Energy expenditure during front crawl swimming: predicting success in middle-distance events. Int. J. Sports Med. 6:266-270. doi: 10.1055/s-2008-1025849

de Mello Vitor, F., and Böhme, M. T. S. (2010). Performance of young male swimmers in the 100-meters front crawl. Pediatr. Exerc. Sci. 22, 278-287. doi: $10.1123 /$ pes.22.2.278

de Morton, N. A. (2009). The PEDro scale is a valid measure of the methodological quality of clinical trials: a demographic study. Aust. J. Physiother. 55, 129-133. doi: 10.1016/S0004-9514(09)70043-1

Dekerle, J., Sidney, M., Hespel, J. M., and Pelayo, P. (2002). Validity and reliability of critical speed, critical stroke rate, and anaerobic capacity in relation to front crawl swimming performances. Int. J. Sports Med. 23, 93-98. doi: 10.1055/s-2002-20125

Denadai, B. S., Greco, C. C., and Teixeira, M. (2000). Blood lactate response and critical speed in swimmers aged 10-12 years of different standards. J. Sports Sci. 18, 779-784. doi: 10.1080/026404100419838

Duché, P., Falgairette, G., Bedu, M., Lac, G., Robert, A., and Coudert, J. (1993). Analysis of performance of prepubertal swimmers assessed from anthropometric and bio-energetic characteristics. Eur. J. Appl. Physiol. Occup. Physiol. 66, 467-471. doi: 10.1007/BF00599623

Durand-Bush, N., and Salmela, J. H. (2002). The development and maintenance of expert athletic performance: Perceptions of world and Olympic champions. J. Appl. Sport Psychol. 14, 154-171. doi: 10.1080/10413200290103473

Ferraz, R., Branquinho, L., Loupo, R., Neiva, H., and Marinho, D. A. (2020). The relationship between anthropometric characteristics and sports performance in national-level young swimmers. Eur. J. Hum. Mov. 45:20. doi: 10.21134/eurjhm.2020.45.2

Ferreira, M. I., Barbosa, T. M., Costa, M. J., Neiva, H. P., and Marinho, D. A. (2016). Energetics, biomechanics, and performance in masters' swimmers: a systematic review. J. Strength Cond. Res. 30, 2069-2081. doi: 10.1519/JSC.0000000000001279

Ferreira, S., Carvalho, D., Monteiro, A. S., Abraldes, J. A. A., VilasBoas, J. P., Toubekis, A., et al. (2019). Physiological and biomechanical evaluation of a training macrocycle in children swimmers. Sports 7:57. doi: $10.3390 /$ sports7030057

Ferreira, S., Carvalho, D. D., Cardoso, R., Rios, M., Soares, S., Toubekis, A., et al. (2021). Young swimmers' middle-distance performance variation within a training season. Int. J. Environ. Res. Public Health. 18:1010. doi: 10.3390/ijerph18031010

Figueiredo, P., Silva, A., Sampaio, A., Vilas-Boas, J. P., and Fernandes, R. J. (2016). Front crawl sprint performance: a cluster analysis of biomechanics, energetics, coordinative, and anthropometric determinants in young swimmers. Mot. Control 20, 209-221. doi: 10.1123/mc.2014-0050

Garrido, N., Marinho, D. A., Barbosa, T. M., Costa, A. M., Silva, A. J., PérezTurpin, J. A., et al. (2010a). Relationships between dry land strength, power variables and short sprint performance in young competitive swimmers. J. Hum. Sport Exerc. 5, 240-249. doi: 10.4100/jhse.201 0.52 .12

Garrido, N., Marinho, D. A., Reis, V. M., van den Tillaar, R., Costa, A. M., Silva, A. J., et al. (2010b). Does combined dry land strength and aerobic training inhibit performance of young competitive swimmers? J. Sports Sci. Med. 9, 300-310. Available online at: https://www.jssm.org/jssm-09-300.xml\%3EFulltext

Geladas, N. D., Nassis, G. P., and Pavlicevic, S. (2005). Somatic and physical traits affecting sprint swimming performance in young swimmers. Int. J. Sports Med. 26, 139-144. doi: 10.1055/s-2004-817862

Hue, O., Antoine-Jonville, S., Galy, O., and Blonc, S. (2013). Anthropometric and physiological characteristics in young Afro-Caribbean swimmers: a preliminary study. Int. J. Sports Physiol. Perform.8, 271-278. doi: 10.1123/ijspp.8. 3.271
Jürimäe, J., Haljaste, K., Cicchella, A., Lätt, E., Purge, P., Leppik, A., et al. (2007). Analysis of swimming performance from physical, physiological, and biomechanical parameters in young swimmers. Pediatr. Exerc. Sci. 19, 70-81. doi: 10.1123/pes.19.1.70

Kjendlie, P. L., Ingjer, F., Madsen, Ø., Stallman, R. K., and Stray-Gundersen, J. (2004a). Differences in the energy cost between children and adults during front crawl swimming. Eur. J. Appl. Physiol. 91, 473-480. doi: 10.1007/s00421-003-1022-0

Kjendlie, P. L., Ingjer, F., Stallman, R. K., and Stray-Gundersen, J. (2004b). Factors affecting swimming economy in children and adults. Eur. J. Appl. Physiol. 93, 65-74. doi: 10.1007/s00421-004-1164-8

Kjendlie, P. L., and Stallman, R. K. (2008). Drag characteristics of competitive swimming children and adults. J. Appl. Biomech. 24, 35-42. doi: 10.1123 /jab.24.1.35

Koopmann, T., Faber, I., Baker, J., and Schorer, J. (2020). Assessing technical skills in talented youth athletes: a systematic review. Sports Med. 50, 1593-1611. doi: 10.1007/s40279-020-01299-4

Lang, M., and Light, R. (2010). Interpreting and implementing the long term athlete development model: English swimming coaches' views on the (swimming) LTAD in practice. Int. J. Sports Sci. Coach. 5, 389-402. doi: 10.1260/1747-9541.5.3.389

Larson, H. K., Young, B. W., McHugh, T. L. F., and Rodgers, W. M. (2019). Markers of early specialization and their relationships with burnout and dropout in swimming. J. Sport Exerc. Psychol. 41, 46-54. doi: 10.1123/jsep.2018-0305

Lätt, E., Jürimäe, J., Haljaste, K., Cicchella, A., Purge, P., and Jürimäe, T. (2009a). Longitudinal development of physical and performance parameters during biological maturation of young male swimmers. Percept. Mot. Skills. 108, 297-307. doi: 10.2466/pms.108.1.297-307

Lätt, E., Jürimäe, J., Haljaste, K., Cicchella, A., Purge, P., and Jürimäe, T. (2009b). Physical development and swimming performance during biological maturation in young female swimmers. Coll. Antropol. 33, 117-122.

Majid, W. K., Jari, H. S., and Noori, R. A. (2019). Performance training for the development of the harmonic ability and its impact on the rapid force and the achievement of (50) meters of breast for junior. J. Glob. Pharma Technol. $11,71-76$.

Malina, R. M. (2010). Early sport specialization: roots, effectiveness, risks. Curr. Sports Med. Rep. 9, 364-371. doi: 10.1249/JSR.0b013e3181fe3166

Marinho, D. A., Amorim, R. A., Costa, A. M., Marques, M. C., Pérez Turpin, J. A., and Neiva, H. P. (2011). "Anaerobic" critical velocity and swimming performance in young swimmers. J. Hum. Sport Exerc. 6, 80-86. doi: 10.4100/jhse.2011.61.09

Marinho, D. A., Barbosa, T. M., Lopes, V. P., Forte, P., Toubekis, A. G., and Morais, J. E. (2020). The influence of the coaches' demographics on young swimmers' performance and technical determinants. Front. Psychol. 11:1968. doi: 10.3389/fpsyg. 2020.01968

Martindale, R. J., Collins, D., and Daubney, J. (2005). Talent development: a guide for practice and research within sport. Question 57, 353-375. doi: 10.1080/00336297.2005.10491862

Mezzaroba, P. V., and Machado, F. A. (2014). Effect of age, anthropometry, and distance in stroke parameters of young swimmers. Int. J. Sports Physiol. Perform. 9, 702-706. doi: 10.1123/ijspp.2013-0278

Mirwald, R. L., Baxter-Jones, A. D., Bailey, D. A., and Beunen, G. P. (2002). An assessment of maturity from anthropometric measurements. Med. Sci. Sports Exerc. 34, 689-694. doi: 10.1249/00005768-200204000-00020

Morais, J. E., Costa, M. J., Forte, P., Marques, M. C., Silva, A. J., Marinho, D. A., et al. (2014b). Longitudinal intra-and inter-individual variability in young swimmers' performance and determinant competition factors. Motriz: Revista de Educação Física. 20, 292-302. doi: 10.1590/S1980-65742014000300008

Morais, J. E., Forte, P., Silva, A. J., Barbosa, T. M., and Marinho, D. A. (2020b). Data modelling for inter- and intra-individual stability of young swimmers' performance: a longitudinal cluster analysis. Res Quart Exerc Sport. 92, 21-33. doi: 10.1080/02701367.2019.1708235

Morais, J. E., Garrido, N. D., Marques, M. C., Silva, A. J., Marinho, D. A., and Barbosa, T. M. (2013a). The influence of anthropometric, kinematic and energetic variables and gender on swimming performance in youth athletes. $J$. Hum. Kinet. 39, 203-211. doi: 10.2478/hukin-2013-0083

Morais, J. E., Jesus, S., Lopes, V., Garrido, N., Silva, A., Marinho, D., et al. (2012). Linking selected kinematic, anthropometric and hydrodynamic 
variables to young swimmer performance. Pediatr. Exerc. Sci. 24, 649-664. doi: 10.1123/pes.24.4.649

Morais, J. E., Lopes, V. P., Barbosa, T. M., Shin-ichiro, M., and Marinho, D. A. (2020a). How does 11-week detraining affect 11-12 years old swimmers' biomechanical determinants and its relationship with $100 \mathrm{~m}$ performance? Sports Biomech. 1-15. doi: 10.1080/14763141.2020.1726998

Morais, J. E., Marques, M. C., Marinho, D. A., Silva, A. J., and Barbosa, T. M. (2014a). Longitudinal modeling in sports: young swimmers' performance and biomechanics profile. Hum. Mov. Sci. 37, 111-122. doi: 10.1016/j.humov.2014.07.005

Morais, J. E., Saavedra, J. M., Costa, M. J., Silva, A. J., Marinho, D. A., and Barbosa, T. M. (2013b). Tracking young talented swimmers: follow-up of performance and its biomechanical determinant factors. Acta Bioeng. Biomech. 15, 129-138. doi: $10.5277 / \mathrm{abb} 130316$

Morais, J. E., Silva, A. J., Marinho, D. A., Lopes, V. P., and Barbosa, T. M. (2017). Determinant factors of long-term performance development in young swimmers. Int. J. Sports Physiol. Perform. 12, 198-205. doi: 10.1123/ijspp.2015-0420

Morais, J. E., Silva, A. J., Marinho, D. A., Marques, M. C., Batalha, N., and Barbosa, T. M. (2016). Modelling the relationship between biomechanics and performance of young sprinting swimmers. Eur. J. Sport Sci. 16, 661-668. doi: 10.1080/17461391.2016.1149227

Morais, J. E., Silva, A. J., Marinho, D. A., Seifert, L., and Barbosa, T. M. (2015). Cluster stability as a new method to assess changes in performance and its determinant factors over a season in young swimmers. Int. J. Sports Physiol. Perform. 10, 261-268. doi: 10.1123/ijspp.2013-0533

Moreira, M. F., Morais, J. E., Marinho, D. A., Silva, A. J., Barbosa, T. M., and Costa, M. J. (2014). Growth influences biomechanical profile of talented swimmers during the summer break. Sports Biomech. 13, 62-74. doi: 10.1080/14763141.2013.865139

Nevill, A. M., Negra, Y., Myers, T. D., Sammoud, S., and Chaabene, H. (2020). Key somatic variables associated with, and differences between the 4 swimming strokes. J. Sports Sci. 37, 787-794. doi: 10.1080/02640414.2020.1734311

Nugent, F. J., Comyns, T. M., and Warrington, G. D. (2017). Quality vs. quantity debate in swimming: perceptions and training practices of expert swimming coaches. J. Hum. Kinet. 57, 147-158. doi: 10.1515/hukin-2017-0056

Ozeker, K. Y., Bilge, M., and Kose, D. S. Y. (2020). The effect of dry-land training on functional strength and swimming performance of 10-12 years old swimmers. Prog. Nutr. 22:e2020028. doi: 10.23751/pn.v22i2-S.10615

Phillips, E., Davids, K., Renshaw, I., and Portus, M. (2010). Expert performance in sport and the dynamics of talent development. Sports Med. 40, 271-283. doi: 10.2165/11319430-000000000-00000

Poujade, B., Hautier, C., and Rouard, A. (2002). Determinants of the energy cost of front-crawl swimming in children. Eur. J. Appl. Physiol. 87, 1-6. doi: 10.1007/s00421-001-0564-2

Poujade, B., Hautier, C. A., and Rouard, A. (2003). Influence de la morphologie, de VO2max et du coût énergetique sur la performance en natation chez de jeunes nageurs. Sci. Sports. 18, 182-187. doi: 10.1016/S0765-1597(03)0 0164-3

Saavedra, J. M., Escalante, Y., Garcia-Hermoso, A., and Dominguez, A. M. (2013). Training volume and performance of young Spanish national and international level swimmers. S. Afr. J. Res. Sport PH. 35, 163-172.

Saavedra, J. M., Escalante, Y., and Rodríguez, F. A. (2010). A multivariate analysis of performance in young swimmers. Pediatr. Exerc. Sci. 22, 135-151. doi: $10.1123 /$ pes.22.1.135

Sammoud, S., Negra, Y., Bouguezzi, R., Hachana, Y., Granacher, U., and Chaabene, H. (2021). The effects of plyometric jump training on jump and sport-specific performances in prepubertal female swimmers. J. Exerc. Sci. Fit. 19, 25-31. doi: 10.1016/j.jesf.2020.07.003

Sammoud, S., Negra, Y., Chaabene, H., Bouguezzi, R., Moran, J., and Granacher, U. (2019). The effects of plyometric jump training on jumping and swimming performances in prepubertal male swimmers. J. Sports Sci. Med. 18, 805-811. Available online at: https://www.jssm.org/jssm-18-805.xml\%3EFulltext

Sammoud, S., Nevill, A. M., Negra, Y., Bouguezzi, R., Chaabene, H., and Hachana, Y. (2018). 100-m Breaststroke swimming performance in youth swimmers: the predictive value of anthropometrics. Pediatr. Exerc. Sci. 30, 393-401. doi: 10.1123/pes.2017-0220

Seffrin, A. D. E., Lira, C. A., Nikolaidis, P. T., Knechtle, B., and Andrade, M. S. (2021). Age-related performance determinants of young swimmers in 100-and 400-m events. J. Sports Med. Phys. Fitness. 1205:56. doi: 10.23736/S0022-4707.21.12045-6

Seifert, L., Button, C., and Davids, K. (2013). Key properties of expert movement systems in sport. Sports Med.43, 167-178. doi: 10.1007/s40279-012-0011-z

Silva, A., Figueiredo, P., Soares, S., Seifert, L., Vilas-Boas, J. P., and Fernandes, R. J. (2012). Front crawl technical characterization of 11-to 13-year-old swimmers. Pediatr. Exerc. Sci. 24, 409-419. doi: 10.1123/pes.24.3.409

Silva, A. F., Figueiredo, P., Seifert, L., Soares, S., Vilas-Boas, J. P., and Fernandes, R. J. (2013). Backstroke technical characterization of 11-13 year-old swimmers. J. Sports Sci. Med. 12:623. Available online at: https://www.jssm.org/jssm-12-623. xml\%3EFulltext

Staub, I., Zinner, C., Bieder, A., and Vogt, T. (2020a). Within-sport specialisation and entry age as predictors of success among age group swimmers. Eur. J. Sport Sci. 20, 1160-1167. doi: 10.1080/17461391.2019.1702107

Staub, I., Zinner, C., Stallman, R. K., and Vogt, T. (2020b). The consistency of performance among age group swimmers over 8 consecutive years. Ger. J. Exerc. Sport Res. 50:123-129. doi: 10.1007/s12662-019-00628-8

Tijani, J. M., Zouhal, H., Rhibi, F., Hackney, A. C., Ben Ounis, O., Saidi, K., et al. (2019). Relationship between anthropometry and stroking parameters of front crawl sprint performance in young swimmers. Medicina dello Sport. 72, 355-365. doi: 10.23736/S0025-7826.19.03427-6

Toubekis, A. G., Tsami, A. P., and Tokmakidis, S. P. (2006). Critical velocity and lactate threshold in young swimmers. Int. J. Sports Med. 27, 117-123. doi: $10.1055 / \mathrm{s}-2005-837487$

Tsalis, G., Toubekis, A. G., Michailidou, D., Gourgoulis, V., Douda, H., and Tokmakidis, S. P. (2012). Physiological responses and stroke-parameter changes during interval swimming in different age-group female swimmers. J. Strength Cond. Res. 26, 3312-3319. doi: 10.1519/JSC.0b013e31824e1724

Vaeyens, R., Gullich, A., Warr, C. R., and Philippaerts, R. (2009). Talent identification and promotion programmes of Olympic athletes. J. Sports Sci. 27, 1367-1380. doi: 10.1080/02640410903110974

Wiersma, L. D. (2000). Risks and benefits of youth sport specialization: perspectives and recommendations. Pediatr. Exerc. Sci. 12, 13-22. doi: $10.1123 /$ pes.12.1.13

Zacca, R., Azevedo, R., Chainok, P., Vilas-Boas, J. P., Castro, F. A. D. S., Pyne, D. B., et al. (2020). Monitoring age-group swimmers over a training macrocycle: energetics, technique, and anthropometrics. J. Strength Cond. Res. 34, 818-827. doi: 10.1519/JSC.0000000000002762

Zamparo, P., Capelli, C., Cautero, M., and Di Nino, A. (2000). Energy cost of frontcrawl swimming at supra-maximal speeds and underwater torque in young swimmers. Eur. J. Appl. Physiol. 83, 487-491. doi: 10.1007/s004210000318

Zamparo, P., Cortesi, M., and Gatta, G. (2020). The energy cost of swimming and its determinants. Eur. J. Appl. Physiol. 120, 41-66. doi: 10.1007/s00421-019-04270-y

Zarzeczny, R., Kuberski, M., Deska, A., Zarzeczna, D., and Rydz, K. (2013). The evaluation of critical swimming speed in 12-year-old boys. Hum. Mov. 14, 35-40. doi: 10.2478/humo-2013-0002

Conflict of Interest: The authors declare that the research was conducted in the absence of any commercial or financial relationships that could be construed as a potential conflict of interest.

The reviewer NDG declared a shared affiliation, with no collaboration, with one of the authors, AS, to the handling editor at the time of the review.

Publisher's Note: All claims expressed in this article are solely those of the authors and do not necessarily represent those of their affiliated organizations, or those of the publisher, the editors and the reviewers. Any product that may be evaluated in this article, or claim that may be made by its manufacturer, is not guaranteed or endorsed by the publisher.

Copyright (c) 2021 Morais, Barbosa, Forte, Silva and Marinho. This is an open-access article distributed under the terms of the Creative Commons Attribution License (CC $B Y)$. The use, distribution or reproduction in other forums is permitted, provided the original author(s) and the copyright owner(s) are credited and that the original publication in this journal is cited, in accordance with accepted academic practice. No use, distribution or reproduction is permitted which does not comply with these terms. 


\section{ACRONYMS}

\section{Anthropometrics}

AFG-arm flexed girth

AG-ankle girth

AL-arm length

APHV-age at peak height velocity

ARG-arm relaxed girth

AS-arm span

AS/H-arm span / height index

BA-body area

BF-body fat

Biacr B-biacromial breadth

Biacr B/Biiliac B-biacromial breadth/biiliac breadth index

Biiliac B-biiliac breadth

Bitroch B-bitrochanteric breadth

Biacr B/H-biacromial breadth/height index

$\mathrm{BL}$-body length

BM-body mass

BMM - bone mineral mass

BMI-body mass index

BSA-body surface area

Calf $\mathrm{G}$-calf girth

CC-chest circumference

$\mathrm{CG}$-chest girth

CG/H - chest girth/height index

$\mathrm{CP}$-chest perimeter

EB-elbow breath

FG-forearm girth

ForL-forearm length

FM-whole body fat

FFM - fat free mass

FL-foot length

FSA-frontal surface area

FW-foot width

GG-gluteal girth

GG/H-gluteal girth/height index

$\mathrm{H}$-height

HL-hand length

Hlift-hydrostatic lift

HSA - hand surface area

HW-hand width

$\mathrm{KB}$-knee breadth

LAL-lower arm length

LBM-lean body mass

LG-leg girth

LL-leg length

LLL-lower limb length

PS-propelling size

$\mathrm{RH}$-reach height

SBMD_spine bone mineral density

$\mathrm{SH}$ - sitting height

SS-subscapular skinfold

SSS-sum of six skinfolds

S9-sum of nine skinfolds

TBMD_total bone mineral density

TG-thigh girth
TL_thigh length

TLEL-total lower extremity length

TS-triciptal skinfold

TSA-thoracic section area

TTSA - trunk transverse surface area

TUEL-total upper extremity length

TW-trunk width

ULL-upper limb length

UAL-upper arm length

WB-wrist breadth

WG-wrist girth

Biomechanics

$\mathrm{AE}$ - arm extension

AFlex-ankle flexibility

AvgPext-average power extension

AvgPflex-average power flexion

AvgPer-average power external shoulder rotation

AvgPir-average power internal shoulder rotation

BE-back extension

BJ-broad jump

$\mathrm{BP}$-bench press

$\mathrm{BR}$ - ball range

BT-ball throwing

$\mathrm{Bu}$-buoyancy

$\mathrm{C}_{\mathrm{DA}}$ - coefficient of active drag

$\mathrm{C}_{\mathrm{Dp}}$-coefficient of passive drag

CMJ-countermovement jump

$\mathrm{D}_{\mathrm{a}}$ - active drag

$\mathrm{D}_{\mathrm{aF}}$-drag factor

$\mathrm{D}_{\mathrm{e}}$-drag efficiency

$\mathrm{D}_{\mathrm{p}}$ - passive drag

$\mathrm{dv}$-intra-cyclic variation of the swim speed

$\mathrm{dv} / \mathrm{v}$-intra-cyclic variation of the swim speed/swim speed index

EExt-elbow extension

EFlex-elbow flexion

$\mathrm{E}_{\text {tot }}$-total power input

$\mathrm{F}_{\mathrm{r}}$-Froude number

Glide-gliding variables

$\mathrm{HG}$-hand grip

HExt-hip extension

HAbd-hip abduction

HJ-horizontal jump

HL-hydrostatic lift

HS-hand slip

IdC-index of coordination

KFE-knee flexion/extension

LE-leg extension

$\mathrm{LF}_{\text {ext }}$-left forearm external rotation

$\mathrm{LF}_{\text {int }}$-left forearm internal rotation

MF-mean force

MMI-mean mechanical impulse

$\mathrm{PC}$ - pronated chin-ups

$\mathrm{P}_{\mathrm{d}}$ - power to overcome drag

$\mathrm{P}_{\mathrm{k}}$-mechanical power to transfer kinetic energy to water

$\mathrm{P}_{\text {ext }}$-external mechanical power

PText-peak torque extension

PTflex-peak torque flexion 
PTer-peak torque external shoulder rotation

PTir-peak torque internal shoulder rotation

$\mathrm{R}_{\mathrm{e}-\text { Reynolds number }}$

$\mathrm{RF}_{\text {ext }}$-right forearm external rotation

$\mathrm{RF}_{\text {int }}$-right forearm internal rotation

SAbd-shoulder abduction

SAdd-scapular adduction

$\mathrm{SF}$-stroke frequency

SFlex-shoulder flexibility

SFlexion-shoulder flexion

SL-stroke length

$\mathrm{SL} \cdot \mathrm{pSL}^{-1}$-stroke length normalized for anatomical potential stroke length

SLJ-standing long jump

SL/AS-stroke length/arm span index

TDI-technique drag index

$\mathrm{v}$-swim speed

$\mathrm{v}_{\mathrm{h}}$-hull speed

VJ-vertical jump

Vol-body volume

$\mathrm{UT}$-underwater torque

25-m KWP-a 25-m kick without a push

25-m free WP-25-m freestyle without a push

$\triangle \mathrm{CM}-\mathrm{CV}$-distance between the center of mass and the center of volume

$\alpha_{63}$-body angle with the water line

Energetics/efficiency

Abd-abdominals test

AnCV-anaerobic critical velocity

AnP-anaerobic power

Bl-blood lactate

$\mathrm{Bg}$-blood glucose

$\mathrm{C}_{\mathrm{s}}$ - energy cost of swimming

$\mathrm{C}_{\mathrm{s}} / \mathrm{SA}$ - energy cost of swimming calculated per unit of surface area

$\mathrm{C}_{\mathrm{s}}$ /SA.HL-energy cost of swimming calculated per unit of surface area and hydrostatic lift

CSR_critical stroke rate

$\mathrm{CV}$-critical velocity

FAH-flexed arms hang

$\mathrm{FB}$-flamingo balance

$\mathrm{eVO}_{2 \max }$ - estimated aerobic power

HR-heart rate

$\mathrm{HR}$ rest-resting heart rate

$\mathrm{MP}_{30}$-mean power in $30 \mathrm{~s}$

MAV-maximal aerobic velocity

$\eta_{\mathrm{F}}$-Froude efficiency

PT-plate tapping

RR sys-resting systolic blood pressure

RR diast-resting diastolic blood pressure

$\mathrm{RPE}$ - rate of perceived exertion

SandR-sit and reach

SI-stroke index

SR-shuttle run

SRE-shuttle run endurance

V4-velocity corresponding to a blood lactate concentration of $4 \mathrm{mmol} \cdot \mathrm{l}^{-1}$
$\mathrm{VO}_{2 \max }$ - maximal oxygen uptake

$\mathrm{VO}_{2 \text { peak }}$ - peak oxygen uptake

$\mathrm{VO}_{2}$-oxygen consumption

$\Delta \mathrm{La}-$ net increase of blood lactate 